\title{
Cálculo y comparación de la prima de un reaseguro de salud usando el modelo de opciones de Black-Scholes y el modelo actuarial ${ }^{*}$
}

\author{
Recibido: Febrero 17, 2015 - Aceptado: Marzo 4, 2016
}

Doi: dx.doi.org/10.12804/rev.econ.rosario.18.02.2015.03

\author{
Luis Eduardo Girón ${ }^{\dagger}$ \\ Ferney Herrera Cruz $\ddagger$
}

\section{Resumen}

La presente investigación pretende calcular y comparar la prima de un reaseguro usando el modelo de opciones de Black-Scholes y el modelo clásico actuarial tradicional. El período de análisis va desde enero de 2011 hasta diciembre de 2012. Los resultados obtenidos muestran que el modelo de Black-Scholes, que se utiliza normalmente para valorar opciones financieras, puede ser también usado para la estimación de primas de reaseguros de salud; y que la prima neta estimada a partir de este modelo se aproxima a las establecidas por el método actuarial, excepto cuando el deducible del reaseguro es muy alto (por encima de \$200.000.000).

* Artículo derivado del proyecto de investigación “Cálculo y comparación de la prima de un reaseguro de salud usando el modelo de opciones de Black-Scholes y el modelo actuarial", desarrollado en el interior del Grupo de Investigación en Desarrollo Regional (GIDR) (categoría B), de la Pontificia Universidad de Cali, y apoyado por el Departamento de Economía de la misma universidad. Periodo de ejecución: abril 2013-enero 2014.

† Estadístico profesional, Universidad del Valle (Cali, Colombia); magíster en Economía Aplicada, Universidad del Valle; PhD (c) en Métodos Matemáticos Aplicados a la Economía y la Empresa, Universidad Nacional de Educación a Distancia (UNED) (Madrid, España). Docente investigador, grupo GIDR, Departamento de Economía, Pontificia Universidad Javeriana (Cali, Colombia). Correo electrónico: legiron@javerianacali.edu.co

‡ Matemático, Universidad Nacional de Colombia (Bogotá, Colombia); magíster en Economía, Pontificia Universidad Javeriana (Bogotá, Colombia). Actuario jefe de Coomeva Sector Salud. Correo electrónico: fherrerac@unal.edu.co

Para citar este artículo: Girón, L. E., \& Herrera Cruz, F. (julio-diciembre, 2015). Cálculo y comparación de la prima de un reaseguro de salud usando el modelo de opciones de BlackScholes y el modelo actuarial. Revista de Economía del Rosario, 18(2), 211-248. Doi: dx.doi. org/10.12804/rev.econ.rosario.18.02.2015.03 
Clasificación JEL: G22, G13.

Palabras clave: modelo clásico actuarial, modelo de Black-Scholes.

\title{
Computation and Comparison of a Health Reinsurance Premium Using the Black-Scholes-Options Model and the Actuarial Model
}

\begin{abstract}
This paper aims to calculate and compare the reinsurance premium using the Black-Scholes options model and the actuarial classical model. The period of analysis goes from January 2011-December 2012. Empirical results reveal that the Black-Scholes, used frequently to value future options, can be used to estimate the health reinsurance premium and that the net premium estimated using this model are in fact very close to the net premium obtained by means of the actual method, except when the re-insurance deductible is very high (above $\$ 200,000,000)$.

JEL classification: G22, G13.

Keywords: classical actuarial model, Black-Scholes model.

\section{Cálculo e comparação do prêmio de um resseguro de saúde usando o modelo de opções de Black-Scholes e o modelo atuarial}

\section{Resumo}

A presente pesquisa pretende calcular e comparar a prima de um resseguro usando o modelo de opções de Black-Scholes e o modelo clássico atuarial tradicional. O período de análise vai desde janeiro de 2011 até dezembro de 2012. Os resultados obtidos mostram que o modelo de Black-Scholes, que se utilizam normalmente para valorar opções financeiras, pode ser também usado para a estimação de prêmios de resseguros de saúde; e que o prêmio neto estimado a partir deste modelo se aproxima às estabelecidas por o método atuarial, exceto quando o deduzível do resseguro é muito alto (por cima de \$200.000.000).

Classificação JEL: G22, G13.

Palavras-chave: modelo clássico atuarial, modelo de Black-Scholes. 


\section{Introducción}

Un plan de medicina prepagada es un convenio mediante el cual las empresas autorizadas para ello, empresas de medicina prepagada (de ahora en adelante EMP), se comprometen a asumir directamente el costo de los servicios médicos asistenciales, mediante el cobro regular de una cuota pagada en forma anticipada por el contratante.

Los costos médicos asistenciales de las EMP representan el $78 \%$ de los ingresos operacionales, y el $8 \%$ corresponde a eventos de alto costo. Dentro de estos servicios, los de mayor participación son: las TAC, las resonancias magnéticas y las UCI, con el $25 \%, 23 \%$ y $22 \%$, respectivamente. Las enfermedades de alto costo con mayor participación son: el cáncer, las cardiovasculares, los trasplantes y las cirugías del sistema nervioso central, con el 13\%, 9\%,3\% y $2 \%$, respectivamente.

Las EMP no funcionan desde el punto de vista legal como un sistema de seguridad social, sino como un contrato de aseguramiento. En este sentido, los usuarios determinan los grados de cobertura y los tipos de beneficios para el afiliado individual o para el afiliado y su grupo familiar.

Uno de los principales problemas que enfrentan estas empresas es el de la evaluación del riesgo en salud (valuación actuarial), y el de encontrar metodologías o modelos matemáticos adecuados que les permitan estimar el costo médico generado por los servicios cubiertos por el plan, esto es, el costo per capita o la prima pura de riesgo. Así las cosas, las EMP enfrentan incertidumbre sobre la demanda de servicios, pues es posible que los costos medios por asegurado se eleven producto de la presencia de enfermedades catastróficas de alto costo. Una estrategia llevada a cabo por la EMP para mitigar el riesgo de presencia de enfermedades de alto costo en los asegurados es el reaseguro, el cual consiste en volver a asegurar el riesgo de que se presente una enfermedad catastrófica con otra firma aseguradora, de tal manera que, en caso de que se presente dicha enfermedad, la firma reaseguradora indemnizará a la EMP. Para tener derecho a esta indemnización, la EMP debe pagar una prima a la compañía reaseguradora; el valor de la prima de reaseguramiento es resultado de la negociación entre la EMP y la reaseguradora, por lo tanto, es necesario que la EMP realice los cálculos de la prima para tener referentes sólidos en la negociación.

Tradicionalmente, los métodos actuariales se han constituido en la herramienta para calcular la prima tanto del seguro que deben pagar las personas a la EMP para protegerse contra el riesgo de contraer enfermedades catastróficas, como del reaseguro que debe pagar la EMP a la compañía aseguradora (Deelstra y Platin, 2014). Sin embargo, existen trabajos en los cuales se muestra la interrelación que existe entre las finanzas modernas y las ciencias actuariales, 
y en algunos de ellos se aplican los derivados para calcular la prima del seguro y del reaseguro; entre ellos se tiene: Embrechts (1996), Qiu, Li, Wang y Wang (2012), Kawamoto (1998), Gerber y Shiu (1994), Embrechts y Meister (1997), Torres y Agudelo, (2001), Phillips, Cummins y Allen (1998), Merton (1977), Brennan y Schwartz (1976), Lam, Chang y Lee (2002), Cummins y Geman (1995) y Chicaíza (2002).

Los derivados financieros se han constituido en una de las técnicas modernas para gestionar el riesgo, los cuales, utilizados adecuadamente, permiten transferir los riesgos a los que se encuentran sometidos los agentes económicos, sin crear riesgos adicionales.

Una opción es un derivado, la cual puede definirse como un contrato que da derecho a su poseedor a comprar (opción call) o vender (opción put) un activo a un precio determinado durante un período o en una fecha prefijada (Lamonthe y Pérez, 2006). Las opciones funcionan como un seguro, pues permiten a su tenedor cubrirse ante fluctuaciones del precio del activo subyacente; para tener este derecho, el tendedor debe pagar una prima, la cual se asemeja a la prima que cobran las compañías de seguro a sus asegurados.

La presente investigación pretende utilizar la teoría de valoración de opciones para el cálculo de la prima del reaseguro en eventos de servicios y/o enfermedades de alto costo, y compararla con la prima obtenida con el método clásico actuarial. Un acercamiento de las primas obtenidas por los dos modelos permitiría incorporar una nueva metodología en el cálculo de la prima de reaseguramiento menos sofisticada con relación a los métodos actuariales. De esta forma, se enriquecerían los modelos de reaseguramiento de enfermedades de alto costo utilizados por las EMP. Aunque el reaseguramiento no es obligatorio para las EMP en Colombia, se constituye en un mecanismo que repercute en la sostenibilidad económica de ellas.

Este documento se compone de cinco secciones, adicionales a esta introducción. En la primera sección, se realiza la revisión de la literatura y el planteamiento del problema. En la segunda, se plantea la metodología utilizada. En la tercera, se presenta el marco conceptual de los reaseguros y la teoría del modelo de Black-Scholes. En la cuarta, se estiman las primas por los dos métodos y se comparan los resultados. Finalmente, se exponen las conclusiones más relevantes de la investigación.

\section{Revisión de literatura y planteamiento del problema}

En diferentes ámbitos de las ciencias actuariales se han desarrollado diversos trabajos que muestran la interrelación entre los seguros, los reaseguros y las 
finanzas, en esta última específicamente los instrumentos derivados, y especialmente las opciones. Entre dichos trabajos se tienen:

Embrechts (1996) presenta una comparación de los mecanismos de valoración de los métodos tradicionales de los seguros y las finanzas. Adicionalmente, el autor muestra la fuerte interrelación existente entre seguros y finanzas a través de las opciones call europeas y las opciones asiáticas.

Qiu, Li, Wang y Wang (2012) estudian el reaseguro catastrófico y agrupan los métodos para determinar la prima de reaseguro de catástrofes en tres categorías, siendo una de ellas el enfoque académico basado en finanzas y teorías actuariales (teoría de valoración de opciones, teoría de valoración bajo arbitraje y teoría de la optimización de la utilidad).

Kawamoto (1998) expone cómo las opciones pueden ser utilizadas como reaseguros, pues estas dan el derecho a comprar límites adicionales de cobertura a un precio especificado dentro del período de tiempo de la póliza.

Embrechts y Meister (1997) resaltan la aparición de los derivados de seguros catastróficos introducidos por el Chicago Board of Trade (СвОт) en 1992 y consideran que los derivados (opciones y futuros) de seguros catastróficos pueden pensarse como instrumentos de reaseguros estandarizados.

A Phillips, Cummins y Allen (1998), partiendo del concepto de que los contratos de seguros son instrumentos financieros, les parece natural aplicar modelos financieros para la fijación de precios de seguros. Bajo este concepto y fundamentados en la teoría de opciones, desarrollan un modelo de valoración financiera para determinar el valor de la prima por línea de una compañía de seguros que posee varios tipos de seguros. Para estos autores, una de las ventajas de los modelos de valoración aplicados en finanzas sobre los modelos actuariales clásicos es que reconocen que los precios de los seguros en lo posible no deben crear oportunidades de arbitraje.

Merton (1977), a partir de la fórmula desarrollada por Black-Scholes para una opción put, deriva el costo de una garantía de pago para un préstamo.

Brennan y Schwartz (1976) determinan el precio de equilibrio de un seguro de vida variable; el beneficio del comprador del seguro se compone de una cantidad segura más otra parte que depende del comportamiento de una opción call sobre un portafolio de referencia. La cantidad garantizada o segura constituye el precio de ejercicio de la opción.

Gerbert y Shiu (1994), utilizando la transformada de Esscher, la cual es una herramienta de las ciencias actuariales, logran derivar, entre otras, la fórmula de valoración de opciones desarrollada por Black-Scholes (1973), mostrando así la interconexión existente entre las finanzas modernas y las ciencias actuariales.

Cummins y Geman (1995) estudian la fijación de precios futuros y de estrategias con opciones financieras de seguros contra catástrofes. Resaltan la 
importancia de estos instrumentos de cobertura introducidos por el Chicago Board of Trade en 1992 y 1993, respectivamente, en la estabilización del mercado de seguros, constituyendo una estrategia de reaseguramiento.

Chicaíza (2003) utiliza las opciones financieras como mecanismo para estimar las primas de seguro y reaseguro en una EPS del sistema de salud colombiano.

La revisión bibliográfica anterior, exceptuando el trabajo de Chicaíza (2002), muestra la poca aplicación de la teoría de opciones para el cálculo de las primas de reaseguramiento en el sector de salud (EMP). Sin embargo, el trabajo de Chicaíza no desarrolla el método actuarial, por lo tanto, no puede comparar sus resultados con los que se obtendrían con la actuaría tradicional. En este sentido, la presente investigación pretende llenar dicho vacío, siendo este el aspecto novedoso del trabajo. Partiendo del concepto de que una opción es un seguro, se plantea como hipótesis de la investigación que la prima de un reaseguro de eventos de alto costo aplicado a una EMP puede ser calculada con la fórmula de valoración de opciones desarrollada por Black-Scholes (1973), y que esta prima se aproxima significativamente a la estimada mediante el modelo actuarial.

\section{Metodología}

El presente trabajo de investigación está fundamentado en la teoría clásica actuarial y en el modelo de Black-Scholes para valoración de opciones. La información utilizada en la elaboración de la presente investigación se generó de la base de datos de los costos médicos asistenciales de una EMP con gran participación en el contexto de salud colombiano. El período de cubrimiento de análisis fue de dos años (2011 y 2012). La base de datos detalla los costos de cada uno de los servicios utilizados por cada afiliado a la EMP. Los archivos se encuentran en formato plano .txt, y los softwares empleados para las estimaciones y los cálculos fueron: Access MS, SPSS y @RISK.

Los pasos seguidos para responder el objetivo propuesto en la investigación son:

1) Depuración de la base de datos, eliminando registros duplicados y servicios con costo cero.

2) Descripción estadística de la información utilizada.

3) Aplicación de la prueba estadística no paramétrica de Kolmogórov-Smirnov (K-S) para demostrar que la información utilizada se comporta de manera similar entre dos años consecutivos. 
4) Identificación de la función de probabilidad que mejor modele la frecuencia de los servicios.

5) Identificación de la función de probabilidad que mejor modele la severidad (costos) de los servicios, con la estimación de los parámetros y prueba de bondad de ajuste ji-cuadrado.

6) Aplicación del modelo actuarial de frecuencia-severidad, con aplicación de SPSS y @RISK.

7) Cálculo de primas puras de riesgo del reaseguro de eventos de alto costo, mediante el modelo empírico determinístico y el modelo actuarial, con varias opciones de deducible.

8) Aplicación del modelo de Black-Scholes (1973) para valoración de opciones, con estimación de la volatilidad mediante información histórica diaria de las cuentas médicas radicadas en la EMP.

9) Cálculo de las primas netas con la estimación del factor de seguridad para cada uno de los modelos utilizados.

10) Comparación de resultados mediante las dos vías: modelo actuarial y modelo de opciones financieras Black-Scholes (1973).

11) Conclusiones y recomendaciones.

\section{Marco de referencia}

En esta sección se presentan los principales elementos de la teoría del reaseguro, el modelo de valoración de opciones Black-Scholes (1973) y la conexión entre la teoría del reaseguro y la valoración de opciones.

\subsection{El concepto de reaseguro}

Al hablar de reaseguro resulta indispensable referirse primero a lo que es el seguro, ya que este es el origen de todo reaseguro. Es por esto que a continuación se mencionan cuáles son los elementos del seguro, y con esto empezar a hacer el análisis de cuáles son los riesgos que una compañía aseguradora, EMP en la presente investigación, asume al aceptar un riesgo, y por la que se puede ver en la necesidad de reasegurarse.

Para que un sistema de seguros sea estable; es decir, para que las utilidades esperadas se cumplan o estén dentro de cierto rango 'aceptable', es recomendable que determinadas características se cumplan. Entre las características más importantes se tienen: 
- Gran número de asegurados: dado que la prima que se cobra se basa en la ley de los grandes números, nuestra confianza respecto al valor esperado del monto de los siniestros aumentará, o será mayor, si la población asegurada es grande.

- Valores a riesgo homogéneos: resulta obvio el hecho de que al tener mayor homogeneidad en las sumas aseguradas en riesgo, menor será la varianza que se tenga respecto al monto total de los siniestros.

- Bases de cálculos actuariales adecuadas: las primas que se cobran deben estar basadas en información que contemple el aspecto dinámico del riesgo por cubrir. Muchas veces, no es posible contar con esta información y se tendrá que hacer una estimación conservadora.

- Documentos contractuales claros: es importante que los documentos contractuales reflejen las bases en las que se calcularon las primas. De no ser así, se podrían estar cubriendo riesgos de forma gratuita, lo que implicaría una alta siniestralidad.

- Buena suscripción: aunque en la práctica es imposible eliminar por completo el riesgo de antiselección, el contar con una buena selección de riesgos ayudará a reducir la antiselección a un nivel aceptable.

\subsubsection{El contrato de reaseguro}

En la práctica existen dos formas de lograr homogeneizar las sumas aseguradas: el coaseguro ${ }^{1}$ y el reaseguro. Dado que el objetivo principal de este trabajo es hablar del reaseguro, se describe en esta sección lo que es el reaseguro, así como algunas modalidades de este.

El reaseguro es una forma aseguradora de segundo grado, a través de cuyas diversas modalidades las entidades aseguradoras (EMP) procuran homogeneizar y limitar las responsabilidades a su cargo para normalizar el comportamiento de la cartera de riesgos asumidos. Esta normalización se da por medio de la cobertura de los desvíos o desequilibrios que afecten la frecuencia, la intensidad, la distribución temporal o la cuantía individual de los siniestros que se produzcan de ella. Visto de manera sencilla, se puede decir que el reaseguro es asegurar lo que ya está asegurado. De esta forma, algunos de los elementos del seguro mencionados en la sección anterior vuelven a reaparecer en el contrato de reaseguro. Así pues, el principio de buena fe toma mayor jerarquía, ya que se trata de un contrato entre 'expertos'.

1 El coaseguro es la concurrencia acordada de dos o más entidades aseguradoras en la cobertura de un mismo riesgo. 
El asegurado (afiliado a la EMP) únicamente mantiene relación contractual con la aseguradora (cedente), y en ningún caso esa relación contractual se puede ver afectada por las relaciones entre la EMP y los reaseguradores que pudieran tener acuerdos con la EMP.

\subsubsection{Modelo actuarial de la prima de reaseguro}

Desde un punto de vista clásico, Lundberg (1940) introduce la teoría de riesgo para analizar la variabilidad inherente a los problemas actuariales. En particular, la teoría de riesgo colectivo analiza los resultados de un portafolio de seguros a partir del monto agregado de siniestros. Esto es razonable, ya que son los siniestros reales los que permiten tomar decisiones.

Se entiende como prima de riesgo aquella en la que el reasegurador refleja el precio básico del riesgo que cubrirá, en función de la probabilidad de ocurrencia y del monto de la pérdida esperada en la que incurrirá por concepto del siniestro. El reaseguro está relacionado con variables aleatorias, tales como el número de siniestros $N$ y los montos de cada siniestro $Z_{i}, i=1, \ldots, N$, las cuales se suponen independientes e idénticamente distribuidas (Bowers et al., 1997).

De la combinación de $N$ y $Z_{i}$ se obtiene la variable aleatoria compuesta del monto agregado de siniestros, que constituye la cantidad total que la EMP pagará en un año, y se representa como

$$
X=\sum_{i=1}^{N} Z_{i}
$$

\subsubsection{Distribución del número de siniestros}

Generalmente, en la práctica no se cuenta con suficientes datos que permitan obtener una distribución que tenga un buen ajuste a los datos observados; sin embargo, es normal suponer que la distribución del número de siniestros se comporte como una distribución de Poisson o binomial negativa. En seguros generales, y principalmente en salud, el número de siniestros se considera asociado a una distribución de Poisson homogénea, la cual tiene la función de densidad de probabilidad:

$$
f(x)=\frac{\lambda^{x} e^{-\lambda}}{x !} \lambda>0, x=0,1,2, \cdots
$$


Donde $f(x)$ es la probabilidad de que ocurran exactamente $x$ siniestros por unidad de tiempo; $y$ es el número esperado de reclamos por unidad de tiempo aproximado a la frecuencia de siniestros.

El supuesto anterior se basa en las siguientes consideraciones:

- Los siniestros son independientes.

- El número de siniestros en un intervalo de tiempo depende solo de la longitud del intervalo.

- La probabilidad de un número infinito de siniestros en un intervalo de tiempo es cero.

\subsubsection{Distribución del monto de los siniestros}

Las distribuciones que se asocian al monto de siniestros, o severidad, son conocidas como distribuciones de pérdida, por sus consecuencias para el reasegurador (Hogg y Klugman, 1979). Dichas distribuciones son sesgadas y con colas superiores pesadas, pero su uso se justifica por el hecho de que existe mayor probabilidad de ocurrencia de siniestros con montos menores y probabilidad de ocurrencia muy pequeña de siniestros con montos grandes. Como posibles distribuciones de los montos de reclamación o costos médicos asistenciales, se proponen las siguientes funciones de probabilidad:

Tabla 1. Distribuciones de probabilidad de montos de los siniestros

\begin{tabular}{l} 
Pearson 5: $f(x)=\frac{\exp (-\beta /(x-\gamma))}{\beta \Gamma(\alpha)((x-\gamma) / \beta)^{\alpha+1}}$ \\
\hline Loglogística: $f(x ; \alpha, \beta)=\frac{(\beta / \alpha)(x / \alpha)^{\beta-1}}{\left[1+(x / \alpha)^{\beta}\right]^{2}}$ \\
\hline Gamma: $f(x)=\lambda e^{-\lambda x \frac{(\lambda x)^{k-1}}{\Gamma(k)}}$ \\
\hline$f(x ; \mu, \sigma)=\frac{1}{x \sigma \sqrt{2 \pi}} e^{-(\ln x-\mu)^{2} / 2 \sigma^{2}}$ \\
Log-normal:
\end{tabular}




Pareto: $f_{X}(x)=\left\{\begin{array}{cc}\alpha \frac{x_{m}^{\alpha}}{x^{\alpha+1}} & \text { si } x>x_{m}, \\ 0 & \text { si } x<x_{m} .\end{array}\right.$
Weibull: $f(x ; \lambda, k)=\left\{\begin{array}{cc}\frac{k}{\lambda}\left(\frac{x}{\lambda}\right)^{k-1} e^{-(x / \lambda)^{k}} \quad x \geq 0 \\ 0 \quad x<0\end{array}\right.$
Burr: $f(x ; c, k)=c k \frac{x^{c-1}}{\left(1+x^{c}\right)^{k+1}}$

Fuente: Mood, Graybill y Boes (1974).

\subsubsection{Prima pura de riesgo}

El modelo general para el cálculo de la prima de riesgo para este seguro se ilustra a continuación:

$$
P=\sum_{n=1}^{\infty} p(n) \int_{0}^{\infty} x S^{* n}(x) d x
$$

En donde: $p(n)$ es la probabilidad de que en un año ocurran exactamente $n$ siniestros; $S(n)$ es la función de densidad de la distribución de la suma de los montos de las reclamaciones o siniestros; $S^{*}(x)$ es la convolución de orden $n$; y $P$ es la prima neta por unidad de tiempo (Berger, 1985; De Vydler, 1996).

El modelo planteado constituye un método para estimar el monto de las primas puras de riesgo de un reaseguro. La aplicación de este modelo se puede extender a cualquier entidad de aseguramiento que haga parte del Sistema General de Seguridad Social en Salud.

\subsection{Modelo de Black-Scholes de opciones financieras}

Las opciones se han convertido en una estrategia de cobertura y se asemejan a un seguro, pues su tenedor tiene el derecho a hacer uso de la opción cuando el precio del activo subyacente en $T, S_{T}$, es superior al precio de ejercicio o pactado $K$. Por el contrario, si el precio del activo subyacente en $T$, $S_{T}$, es igual o inferior al precio de ejercicio o pactado, su tenedor no ejercerá la opción y la 
opción vencerá sin valor alguno (Marín y Rubio, 2001); en resumen, los pagos de la call europea al vencimiento pueden resumirse como

$$
\begin{aligned}
& S_{T^{-}} K \text { si } S_{T}>K \\
& \text { O si } S_{T}<K
\end{aligned}
$$

El valor de una opción se denomina prima y se asemeja a la prima de riesgo calculada por los métodos tradicionales actuariales; la fórmula más popularizada en tiempo continuo para valorar opciones está fundamentada en el modelo de Black-Scholes (1973), en el cual se asume que:

1) el mercado funciona sin fricciones o sin costos de transacción; 2) los activos son perfectamente divisibles; 3 ) las transacciones se realizan en forma continua y es posible efectuar compras y ventas al descubierto; 4) no hay oportunidades de arbitraje, es decir, no es posible obtener ganancias con una inversión igual a cero; 5) las opciones son europeas y el activo no paga dividendos; 6) la tasa de interés de corto plazo es conocida y constante a lo largo del contrato; 7) los precios del activo siguen una distribución log-normal, lo que implica que sus rendimientos siguen una distribución normal, siendo su volatilidad constante a lo largo del contrato.

El escenario de los supuestos del modelo Black-Scholes son los mercados completos; entendiendo como tales aquellos donde existe solo una medida martingala local equivalente o medida de riesgo neutral, lo que garantiza que no haya en el mercado oportunidades de arbitraje (Guyon y Labordère, 2014).

El cálculo de la prima de una opción bajo ausencia de arbitraje puede realizarse a través de dos métodos:

1) Conformando un portafolio con activos existente en el mercado que replique los pagos del activo que deseamos valorar $\mathrm{y}$, una vez disponible dicha cartera, obtener su costo, que será, en último término, el valor del activo que buscamos valorar.

2) Utilizar las probabilidades neutrales al riesgo y emplear la ecuación fundamental de valoración de activos (Marín y Rubio, 2001).

Aunque los dos métodos producen resultados idénticos en términos del precio del activo, la ventaja de utilizar las probabilidades neutrales al riesgo es que no es necesario conformar una cartera cada vez que se vaya a valorar un activo financiero y que dichas probabilidades pueden extraerse de los precios de mercado de los activos que se negocian. 


\subsubsection{La fórmula de Black-Scholes}

A partir de los supuestos anteriores y asumiendo que el precio del activo es modelado por el siguiente movimiento browniano geométrico

$$
d S=\mu * S * d_{t}+\sigma * S * d W
$$

Donde: $\mu$ es la tasa de retorno esperada, $\sigma$ es la volatilidad del retorno del activo y $d W$ es un movimiento browniano, Black-Scholes (1973), haciendo uso del cálculo estocástico de Ito y de las ecuaciones diferenciales estocásticas parciales, deriva las siguientes fórmulas para valorar el precio teórico de una opción call y una opción put europea cuando la acción no paga dividendos (Shreve, 2004).

$$
\begin{gathered}
\text { CALL: } c=\left[S_{0} \times N\left(d_{1}\right)\right]-\left[E \times e^{-r T} \times N\left(d_{2}\right)\right] \\
\text { PUT: } p=\left[E \times e^{-r T} \times N\left(-d_{2}\right)\right]-\left[S_{0} \times N\left(-d_{1}\right)\right]
\end{gathered}
$$

Donde:

$$
\begin{aligned}
& d_{1}=\frac{\operatorname{Ln}\left(S_{0} / E\right)+\left[\left(r+\sigma^{2} / 2\right) \times T\right]}{\sigma \times \sqrt{T}} \\
& d_{2}=\frac{\operatorname{Ln}\left(S_{0} / E\right)+\left[\left(r-\sigma^{2} / 2\right) \times T\right]}{\sigma \times \sqrt{T}}
\end{aligned}
$$

Las variables necesarias para la aplicación del modelo de Black-Scholes (1973) son: $S_{0^{\prime}}$ precio del activo subyacente en el momento de la valoración de la opción; $E$, el precio de ejercicio; $r$, la tasa de interés en tiempo continuo; $T$, plazo de ejercicio en años; $\sigma$, la volatilidad de la variación del precio del subyacente en términos anuales; $c$, la prima de la opción de compra; y $p$, la prima de la opción de venta (Lamothe y Pérez, 2006).

\subsection{Similitudes entre la teoría de opciones y seguros}

Como lo plantea Chicaíza (2002), en la tabla 2 se muestran las similitudes entre las opciones y los seguros. 
Tabla 2. Similitudes entre las teorías de opciones y seguros

\begin{tabular}{lll}
\hline \multicolumn{1}{c}{ Similitudes } & \multicolumn{1}{c}{ Opciones } & \multicolumn{1}{c}{ Seguros } \\
\hline Cobertura & $\begin{array}{l}\text { Cubren el riesgo de pérdidas eco- } \\
\text { nómicas ante fluctuaciones en el } \\
\text { precio del activo. }\end{array}$ & $\begin{array}{l}\text { Cubren el riesgo de pérdidas eco- } \\
\text { nómicas derivadas de un siniestro. }\end{array}$ \\
Prima & $\begin{array}{l}\text { Volatilidad, variable fundamental } \\
\text { para determinar su valor. }\end{array}$ & $\begin{array}{l}\text { Frecuencia de ocurrencia del su- } \\
\text { ceso, variable fundamental para } \\
\text { determinar su valor. }\end{array}$ \\
Indemnización & $\begin{array}{l}\text { Pago que se debe realizar al tenedor } \\
\text { de la opción en caso de ejercerla. }\end{array}$ & $\begin{array}{l}\text { Pago que se debe realizar al tene- } \\
\text { dor del seguro en caso de que se } \\
\text { presente el siniestro. }\end{array}$ \\
Período del contrato & Por lo general de corto plazo. & \begin{tabular}{l} 
Por lo general de corto plazo. \\
\hline
\end{tabular}
\end{tabular}

Fuente: elaboración de los autores.

Teniendo en cuenta las similitudes anteriores, se espera que el valor de la prima pagada por una opción deba ser el mismo pagado por un seguro bajo las mismas condiciones para que no se genere arbitraje.

En el contexto de las EMP, la equivalencia en las primas permite utilizar la teoría de valoración de opciones para determinar la prima que deben pagar a las compañías reaseguradoras a fin de cubrir el riesgo de que se presenten enfermedades catastróficas, y es en este escenario de las catástrofes que más se ha avanzado en la aplicación de los derivados de seguros catastróficos, como se muestra en la revisión de literatura.

\subsubsection{Equivalencias en la utilización de opciones y seguros para la cobertura de riesgos}

Relacionando las variables involucradas en la fórmula para valorar una opción derivada por Black-Scholes (1973), al reaseguramiento, resulta evidente que el precio de ejercicio es igual al deducible pactado con el reasegurador. Este deducible marca el precio máximo que la EMP tendrá que pagar por la atención de un afiliado. También, el plazo hasta el vencimiento resulta evidente. Este plazo es de un año, ya que el reaseguro se subscribe al inicio de un período anual y el derecho a la indemnización se puede ejercer, en su caso, solo al final de dicho período. El tipo de interés por aplicar tampoco ofrece ninguna dificultad, pues es el vigente para activos 'libres de riesgo' en el momento de la valoración de la opción para el plazo requerido (Petersen y Thiagarajan, 2000). Por su parte, el precio del activo subyacente es la variable más compleja, y esta se debe definir individualmente para cada uno de los afiliados a la EMP, dado que el reaseguro y, por lo tanto, la adquisición de opciones de compra se realizan de forma individual. 
De este modo, el valor del activo subyacente para cada uno de los afiliados será igual al costo que dicho afiliado lleve acumulado en el momento de la valoración de la prima. Este acumulado corresponde al período anual inmediatamente anterior a dicha fecha y será el valor del subyacente por considerar. Para el ejercicio, se toman solamente aquellos afiliados que hayan generado costos, es decir, aquellos registros que tengan valores mayores de cero estrictamente. Si se tomaran aquellos afiliados con costos iguales a cero, la aplicación de la fórmula de Black-Scholes arrojaría un valor indeterminado, pues la fórmula incluye la función logaritmo del cociente entre el valor del activo subyacente y el precio de ejercicio:

$$
\operatorname{Ln}\left(S_{0} / E\right) \text { y } \operatorname{Ln}(0 / E)=\operatorname{Ln}(0)=-\infty
$$

En las opciones financieras existen unos límites que también aplican para el reaseguramiento:

- El precio de una opción de compra nunca puede ser mayor que el valor del activo subyacente, $c<S_{0}$. Nadie va a estar dispuesto a pagar por un derecho de compra más de lo que vale el activo que le da derecho a comprar. Si no se cumple este límite, es decir, si $c \geq S_{0}$, entonces se podría hacer arbitraje. En seguros, el precio de la prima no podrá ser mayor o igual al valor asegurado.

- Nadie va a estar dispuesto a adquirir una obligación (vender una call) a cambio de nada, $c<0$. En seguros, todo riesgo tiene una prima mayor que cero.

- Una opción de compra tiene mayor valor cuando más bajo se dé el derecho a comprar. Si $E_{B}<E_{A}$ entonces $c(E)_{B}<c\left(E_{A}\right)$. En seguros se tiene que, en la medida que aumente el deducible, el valor de la prima baja.

Para cada afiliado, la EMP comprará una opción europea con vencimiento a un año, con precio de ejercicio $E$, que le cubrirá frente a la contingencia de que dicho afiliado genere a lo largo del próximo año unos costos superiores al deducible o precio de ejercicio de la opción. La opción aplicada a la presente investigación debe ser europea, pues el valor total por reclamar al reaseguro se conocerá solamente al final de la vigencia, y en ningún momento anterior a esta. En la práctica, la EMP informa al reasegurador mediante un aviso en el momento en que esta identifique que el afiliado ya ha superado el deducible de la póliza, para que la reaseguradora constituya las respectivas reservas técnicas.

Ejercer la opción antes del vencimiento del término elegido constituye una opción americana, lo que implicaría pérdidas para la EMP originadas por costos 
médicos que el afiliado puede tener posteriormente durante el transcurso de la vigencia, relacionados o por continuidad de la patología base.

Si se presentan riesgos catastróficos o enfermedades de alto costo, el costo medio por paciente se incrementará hasta alcanzar niveles superiores al deducible (E). Suponiendo que el costo medio por paciente se ubique en un nivel igual a un valor $S_{0}$ por encima de $E$, es decir, $S_{0}>E$, la firma de aseguramiento ejercerá su derecho de compra; tiene derecho a comprar el subyacente (costo medio por paciente) a $E$, mientras que el valor de mercado de dicho subyacente es de $S_{0}$. Esto es, si la call se liquida por diferencias, el vendedor de la opción (reasegurador) tendrá que pagar al comprador una cantidad igual a la diferencia entre el precio de mercado y el precio de ejercicio: $\left(S_{0}-E\right)$. Esto indica que la firma de aseguramiento queda resarcida del sobrecosto que le supone el siniestro (el riesgo catastrófico), y paga un costo medio por paciente igual a $E$.

Ahora bien, en el caso de que no se produzca este evento de alto costo, el costo medio por paciente va a situarse en un nivel igual o inferior a $E$. Supóngase, por ejemplo, que dicho costo se sitúa en $S_{i}$, tal que $S_{i} \leq E$ En estas condiciones, la firma de aseguramiento no ejercerá su opción: tiene derecho a comprar a un precio igual a $E$, cuando en el mercado lo puede encontrar más barato $\left(\right.$ a $\left.S_{i}\right)$ (Chicaíza, 2002). Adicionalmente, para la aplicación del modelo se siguen las siguientes hipótesis:

1) La volatilidad es la misma para todas las cuentas de los afiliados.

2) Se asume que los valores promedio de los afiliados y la distribución de acumulados de las cuentas es constante entre dos períodos consecutivos. Se demostrará en el numeral 4.1.

\subsubsection{El problema de la volatilidad}

Una de las variables fundamentales utilizadas en la valoración de las primas de opciones es la volatilidad del rendimiento de $S_{0}$. Para comprenderla, es importante entender los diferentes tipos de volatilidades y las relaciones entre ellas.

En general, la volatilidad mide el riesgo de un activo subyacente y suele calcularse mediante la desviación típica diaria de los rendimientos de los precios. Algunos autores se refieren a la volatilidad como la velocidad de los movimientos de $S_{0}$. Si los precios de $S_{0}$ no se mueven con la suficiente rapidez, las opciones sobre dicho $S_{0}$ valdrán poco dinero, ya que las posibilidades de que el mercado cruce los precios de ejercicio de las opciones son menores. Cuanta mayor volatilidad tenga $S_{0}$, el rango de precios al vencimiento de la opción será mayor, lo que implica un riesgo superior para los vendedores de opciones y mayores probabilidades de beneficio para los compradores de opciones. El 
mercado de opciones traducirá los aumentos de volatilidad en incrementos de precios y a la inversa.

Como la volatilidad futura es desconocida, se tiene que mirar la histórica y predecir volatilidades para ayudar a hacer la mejor estimación sobre el futuro (Demeterfi, 1999). En el análisis final, es la volatilidad futura la que determina el valor de una opción. Para la presente investigación, la volatilidad se expresa anualizada y en porcentaje. Para calcularla, lo ideal sería encontrar una por cada afiliado, para lo cual se debería contar con series históricas de costos de cada uno de los afiliados; situación que es muy difícil de obtener dada la calidad de la información con antigüedad de más de cinco años y por la alta rotación de la población en la EMP. Por esta razón, se adopta la metodología de cálculo utilizada por Chicaíza (2003), que sigue el siguiente proceso:

1) Se calcula la variación diaria de la facturación total de todas las cuentas médicas asistenciales radicadas en la EMP, mediante la siguiente fórmula: $r_{j}=\operatorname{Ln}\left(F_{j} / F_{j-1}\right)$; donde $r_{j}$ es el retorno bruto diario continuamente compuesto del día $j-1$ al día $j ; F_{j}$ es el monto total de la facturación radicada en el j-ésimo día. La ventaja de utilizar esta función para calcular el beneficio es que refleja el interés continuo de los precios. En las siguientes figuras se puede observar el comportamiento de la serie de los $F_{j}$ (figura 1) y de $\operatorname{los} r_{j}$ (figura 2).

2) Luego se calcula la desviación estándar de los $r_{j}$ para el período analizado, notado como $\sigma_{d}$. Dado que el mercado de opciones utiliza la volatilidad anual, se calcula $\sigma=\sigma_{d} \times \sqrt{n}$, en donde $n$ es el número total de días del año en los que la EMP recibió facturación de las IPS.

En otras palabras, la mejor manera de estimar la volatilidad es a partir de la volatilidad histórica, medida como la desviación típica de los datos históricos diarios, multiplicada por la raíz cuadrada del número de días en que las IPS radicaron cuentas asistenciales en la EMP durante un año. Para esto se tiene en cuenta que se requiere una volatilidad anualizada. Al asumir que la distribución de acumulados no varía entre dos períodos consecutivos, se está asumiendo que el costo de los nuevos afiliados a la EMP se va a distribuir de la misma manera a como se hace con los afiliados actuales.

\section{Análisis de resultados}

Los contratos de reaseguro tienen un deducible y, en ocasiones, un tope. El deducible es el valor hasta el cual paga el asegurado, y a partir del cual el costo 


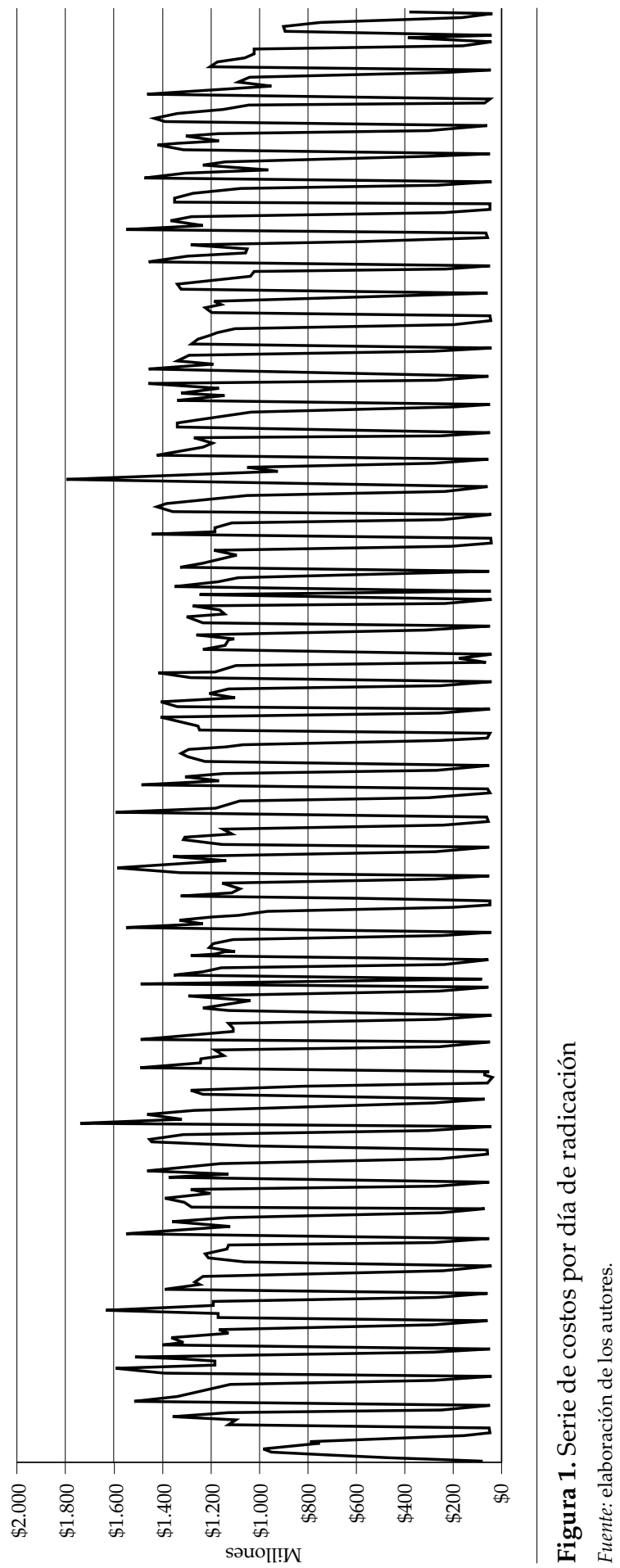




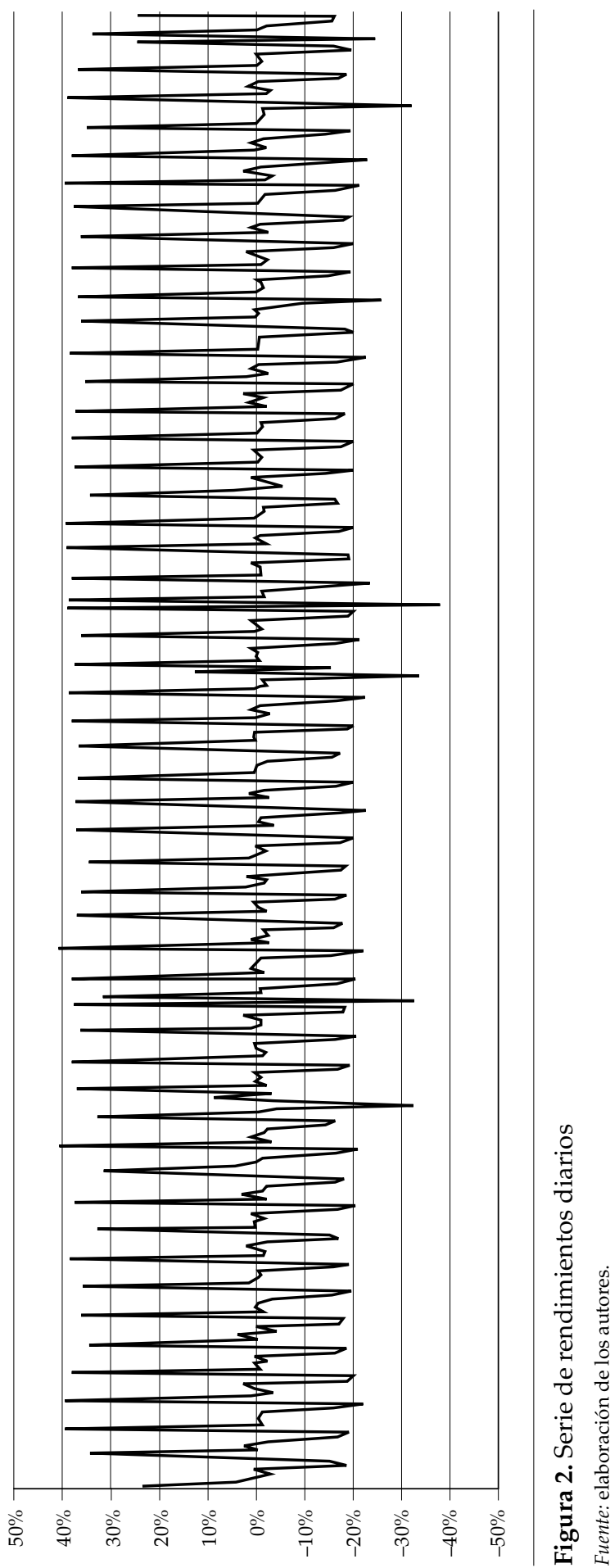


corre por cuenta del reasegurador. El tope es el valor a partir del cual el costo vuelve a correr por cuenta del asegurado, y hasta donde se compromete el reasegurador a pagar una indemnización.

El método de burning cost aplicado a la tarifación de seguros, y fundamentado en información histórica, asume que el comportamiento de los siniestros observados en el pasado sirve como estimador para el comportamiento esperado de estos en el futuro. Se adopta esta metodología para aplicar el modelo actuarial. Se utiliza información del año 2012 para estimar las primas del año 2013. Para tal efecto, se demuestra en el siguiente apartado que los valores promedio y la distribución de acumulados de los costos de la EMP son constantes entre dos períodos consecutivos.

El tipo de reaseguro por utilizar para esta clase de riesgos es no proporcional por exceso de pérdida por riesgo a un año. Mediante esta cobertura el reasegurador se obliga a participar en un siniestro siempre y cuando este supere el deducible establecido previamente.

\subsection{Análisis descriptivo de la información utilizada}

A continuación, se describe la información utilizada de los años 2011 y 2012. Como se observa en las tablas 3 y 4 , la distribución de los pacientes según el costo promedio paciente-año es similar para los dos años. Se destaca que un pequeño porcentaje de la población asegurada consume la mayor cantidad del costo, o, en otras palabras, son los que presentan mayores reclamaciones. Por ejemplo, en la EMP tomada como referencia, solamente el 0,9\% de los afiliados consume el $20 \%$ del total de costos en salud; el promedio de estos afiliados siniestrados fue de \$33 millones.

Tabla 3. Quintiles de los siniestros reales 2011

\begin{tabular}{ccccccc}
\hline Quintil & Pacientes & $\begin{array}{c}\% \text { part. } \\
\text { pacientes }\end{array}$ & Total siniestros & Límite inferior & Límite superior & $\begin{array}{c}\text { Costo } \\
\text { promedio } \\
\text { paciente año }\end{array}$ \\
\hline I & 1.707 & 0,9 & $\$ 56.794 .733 .323$ & $\$ 14.550 .957$ & & $\$ 33.271 .666$ \\
II & 6.843 & 3,5 & $\$ 56.796 .834 .229$ & $\$ 5.630 .153$ & $\$ 14.550 .956$ & $\$ 8.299 .990$ \\
III & 14.451 & 7,5 & $\$ 56.798 .692 .175$ & $\$ 2.826 .231$ & $\$ 5.630 .152$ & $\$ 3.930 .433$ \\
IV & 31.041 & 16,0 & $\$ 56.796 .021 .141$ & $\$ 1.205 .621$ & $\$ 2.826 .230$ & $\$ 1.829 .710$ \\
V & 139.519 & 72,1 & $\$ 56.797 .939 .303$ & & $\$ 1.205 .620$ & $\$ 407.098$ \\
Total & $\mathbf{1 9 3 . 5 6 1}$ & $\mathbf{1 0 0 , 0}$ & $\$ \mathbf{2 8 3 . 9 8 4 . 2 2 0 . 1 7 1}$ & & & $\$ \mathbf{1 . 4 6 7 . 1 5 6}$ \\
\hline
\end{tabular}

Fuente: elaboración de los autores. 


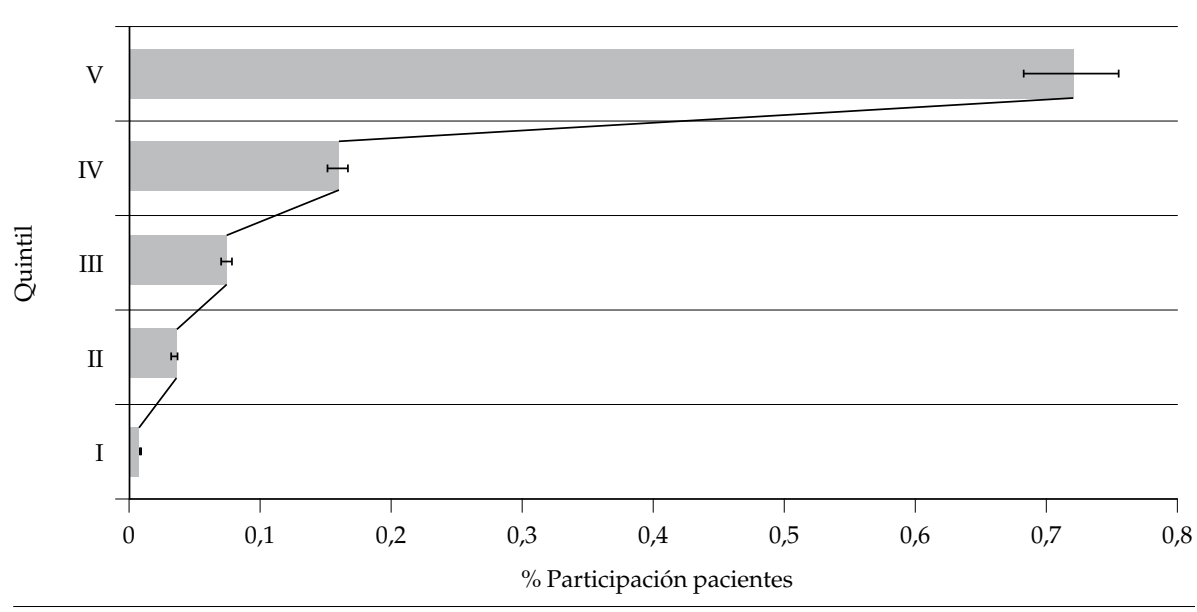

Figura 3. Distribución de quintiles del costo 2011

Fuente: elaboración de los autores.

Tabla 4. Quintiles de los siniestros reales 2012

\begin{tabular}{ccccccc}
\hline Quintil & Pacientes & $\begin{array}{c}\% \text { part. } \\
\text { pacientes }\end{array}$ & Total siniestros & $\begin{array}{c}\text { Límite } \\
\text { inferior }\end{array}$ & $\begin{array}{c}\text { Límite } \\
\text { superior }\end{array}$ & $\begin{array}{c}\text { Costo promedio } \\
\text { paciente año }\end{array}$ \\
\hline I & 1.895 & 0,9 & $\$ 62.969 .285 .045$ & $\$ 14.595 .963$ & & $\$ 33.229 .174$ \\
II & 7.431 & 3,7 & $\$ 62.986 .047 .772$ & $\$ 5.869 .036$ & $\$ 14.595 .962$ & $\$ 8.476 .120$ \\
III & 15.310 & 7,6 & $\$ 62.982 .453 .449$ & $\$ 2.967 .871$ & $\$ 5.869 .035$ & $\$ 4.113 .811$ \\
IV & 32.540 & 16,1 & $\$ 62.982 .170 .336$ & $\$ 1.286 .749$ & $\$ 2.967 .870$ & $\$ 1.935 .531$ \\
V & 144.330 & 71,6 & $\$ 62.980 .593 .053$ & & $\$ 1.286 .748$ & $\$ 436.365$ \\
Total & $\mathbf{2 0 1 . 5 0 6}$ & $\mathbf{1 0 0 , 0}$ & $\$ \mathbf{3 1 4 . 9 0 0 . 5 4 9 . 6 5 5}$ & & & $\$ \mathbf{1 . 5 6 2 . 7 3 5}$ \\
\hline
\end{tabular}

Fuente: elaboración de los autores.

Sin embargo, como se mencionó anteriormente, se debe demostrar formalmente que los valores promedio y la distribución de acumulados de los costos de la EMP son constantes entre dos períodos consecutivos. Para ello, se hará un análisis gráfico de cajas y bigotes, que se contrastará con una prueba estadística no paramétrica.

La figura 5 muestra un alto grado de coincidencia entre las cajas de ambos años, por lo que a priori se puede afirmar que estadísticamente no existen diferencias significativas entre los promedios de dichos períodos. Para validar esta afirmación, se utiliza la prueba estadística no paramétrica de KolmogórovSmirnov (K-S) (Chicaíza, 2003). 


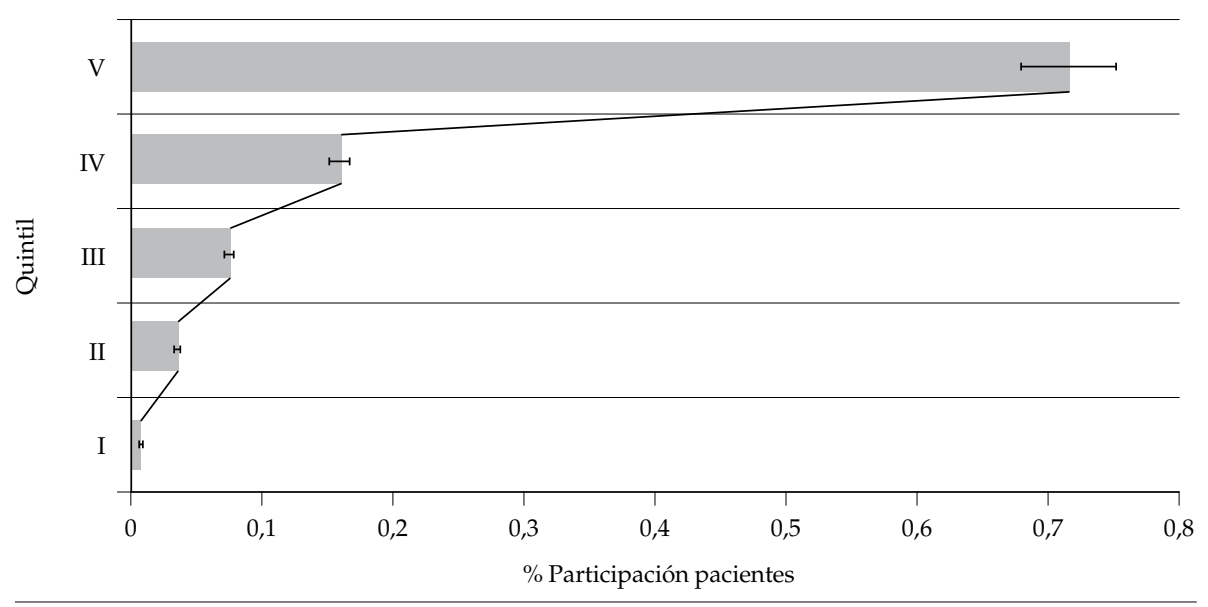

Figura 4. Distribución de quintiles del costo 2012

Fuente: elaboración de los autores.

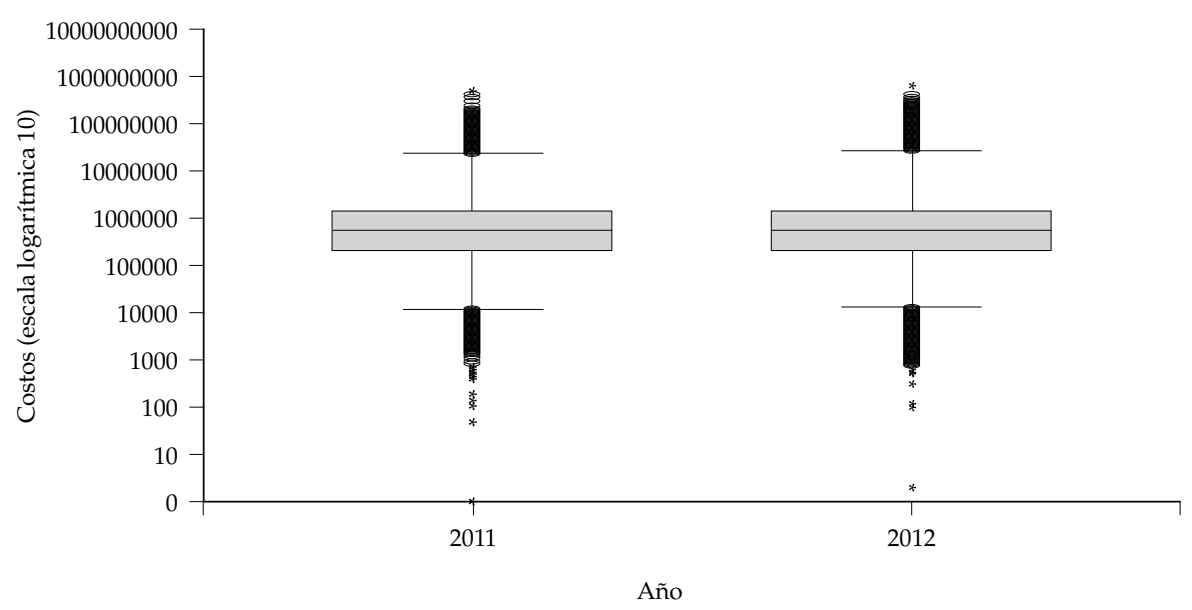

Figura 5. Diagrama de cajas y bigotes costos 2011 y 2012

Fuente: elaboración de los autores empleando el programa SPSs.

La hipótesis nula es de igualdad entre funciones de distribución, y la hipótesis alterna dice que la función de distribución acumulada del año 2011 se comporta de manera diferente a la función de distribución acumulada del año 2012. Formalmente:

$$
\begin{aligned}
& H_{0}: F(x)=G(x) \\
& H_{a}: F(x) \neq G(x)
\end{aligned}
$$


El estadístico por calcular para esta prueba es $(D+m, n)^{2}$, donde $(D+m, n)$ es el supremo de la diferencia entre ambas distribuciones:

$$
(D+m, n)=\operatorname{Sup}(F(x)-G(x))
$$

En donde $m$ y $n$ son los tamaños muestrales.

Al aplicar la prueba en el software SPSS, arrojó que al 0,05 de significancia la hipótesis nula no se puede rechazar, y, por lo tanto, la distribución de costos es la misma entre los dos años.

Tabla 5. Salida SPSS prueba K-S de dos muestras

\begin{tabular}{ccccc}
\hline \multicolumn{4}{c}{ Resumen de prueba de hipótesis } \\
\hline Hipótesis nula & Test & Sig. & Decisión \\
\hline $\begin{array}{l}\text { La distribución de costos es la mis- } \\
\text { ma entre las categorías de año. }\end{array}$ & $\begin{array}{l}\text { Prueba Kolmogórov-Smirnov } \\
\text { de muestras independientes }\end{array}$ & $0,247 \begin{array}{l}\text { Retener la hipó- } \\
\text { tesis nula. }\end{array}$ \\
\hline
\end{tabular}

Se muestran las significancias asintóticas. El nivel de significancia es 0,05.

Fuente: elaboración de los autores empleando el programa SPSS.

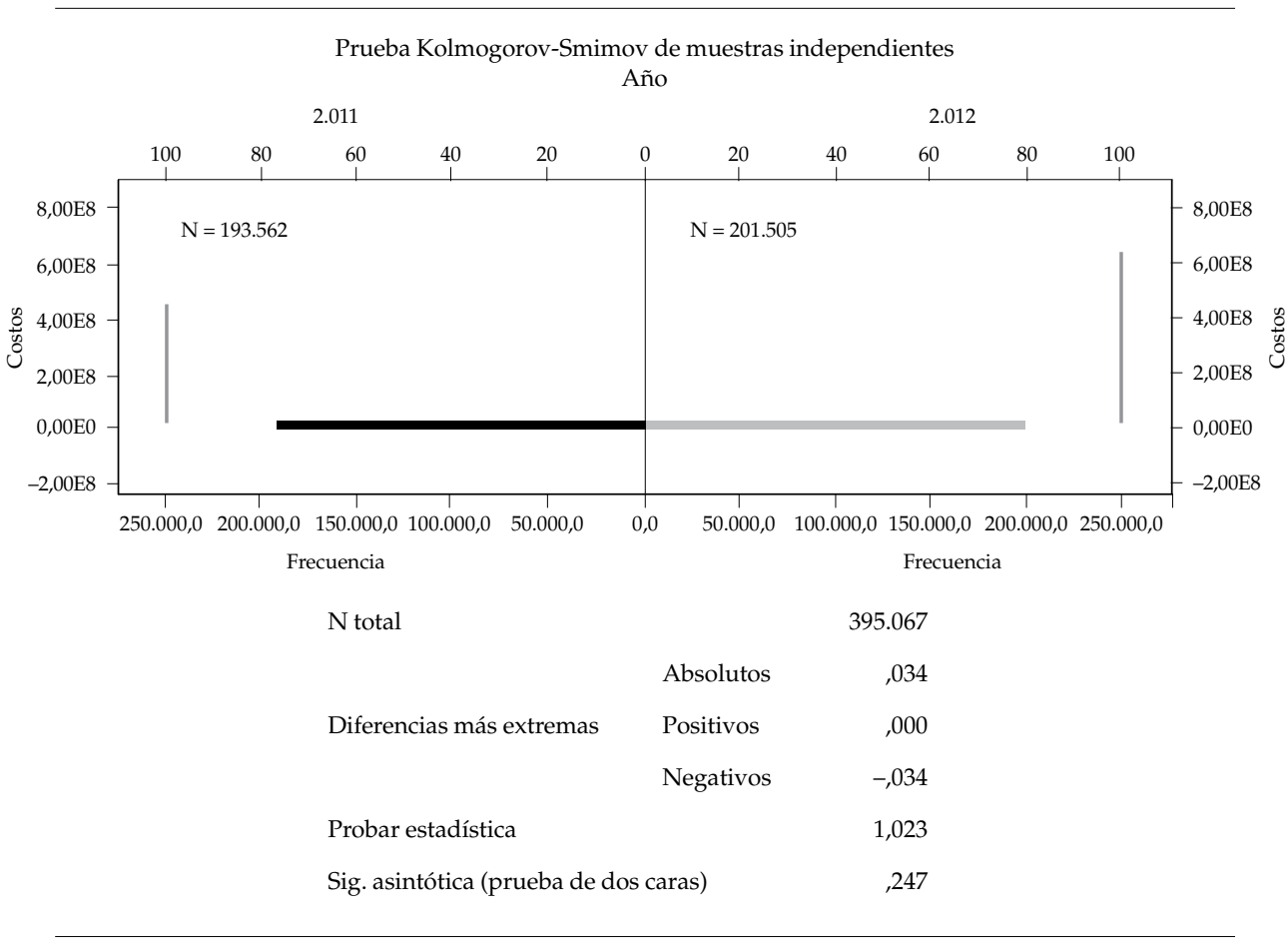

Figura 6. Salida SPSS prueba K-S de dos muestras

Fuente: elaboración de los autores empleando el programa SPSS. 


\subsection{Resultados del modelo actuarial}

A continuación se muestran los resultados obtenidos mediante el software @RISK aplicado a la serie de datos utilizada (201.505 registros). Se presentan las distribuciones de probabilidad estimadas, tanto para el número de reclamaciones o siniestros como para el monto de estas.

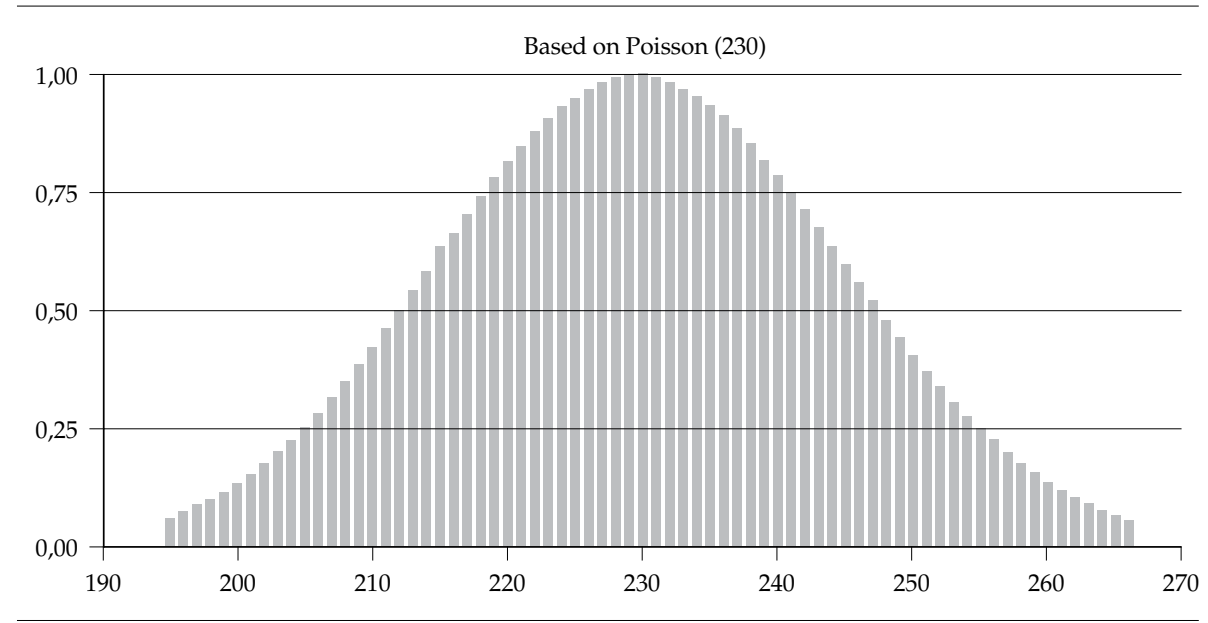

Figura 7. Distribución de número de reclamaciones con deducible $\$ 50.000 .000$ Fuente: elaboración de los autores empleando el programa @RISK.

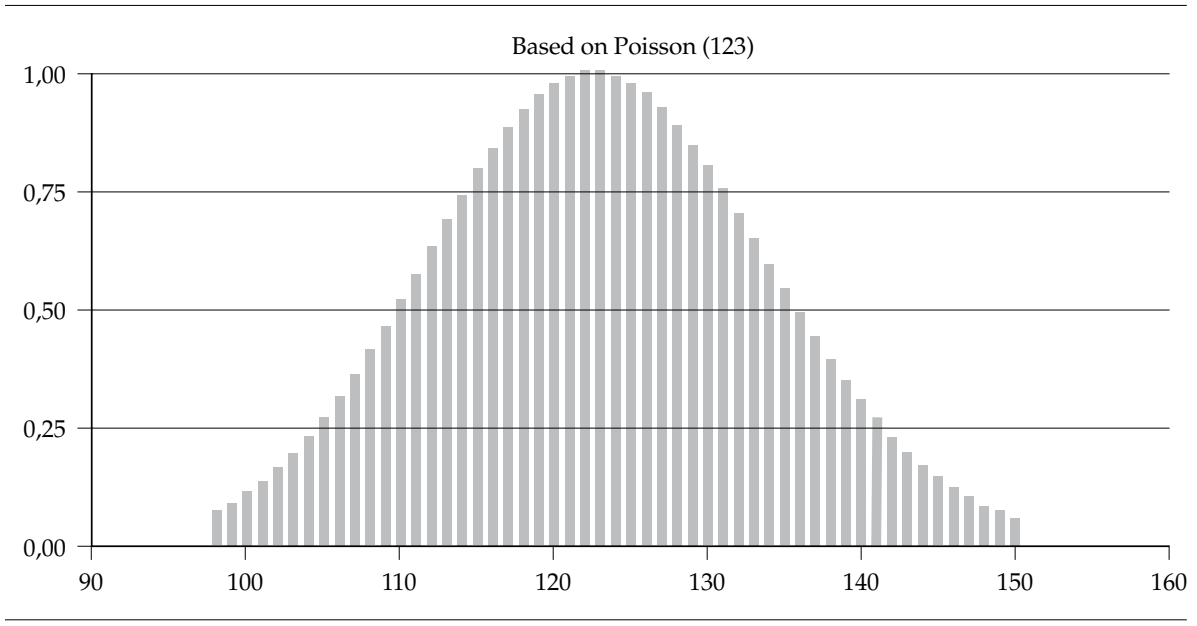

Figura 8. Distribución de número de reclamaciones con deducible $\$ 75.000 .000$

Fuente: elaboración de los autores empleando el programa @RISK. 


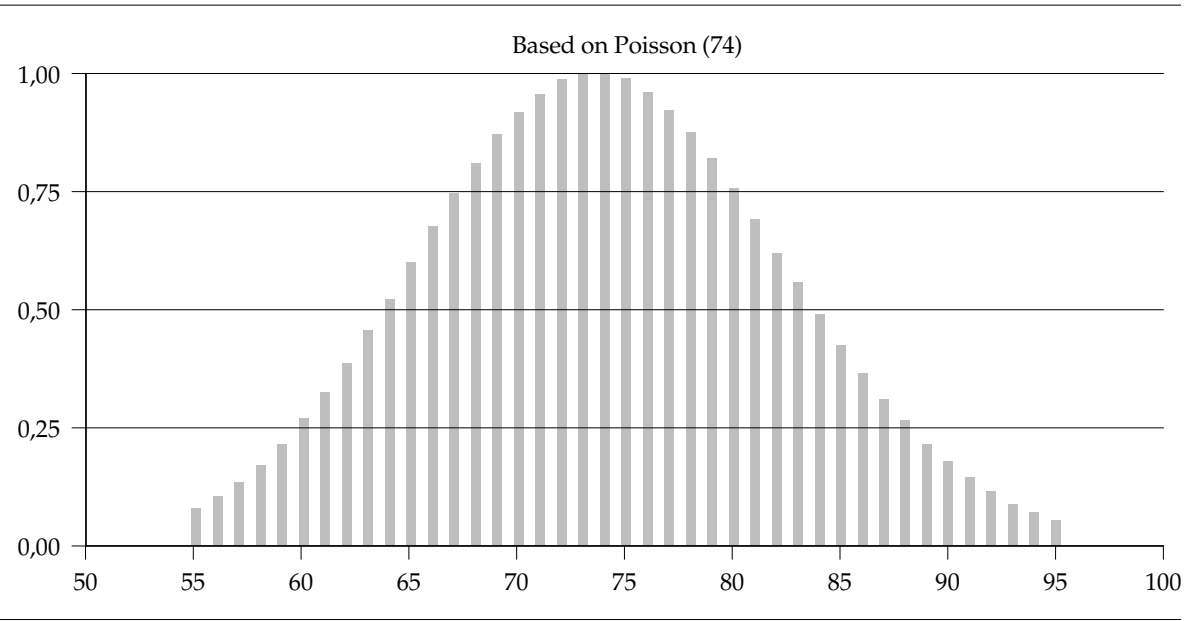

Figura 9. Distribución de número de reclamaciones con deducible $\$ 100.000 .000$ Fuente: elaboración de los autores empleando el programa @RISK.

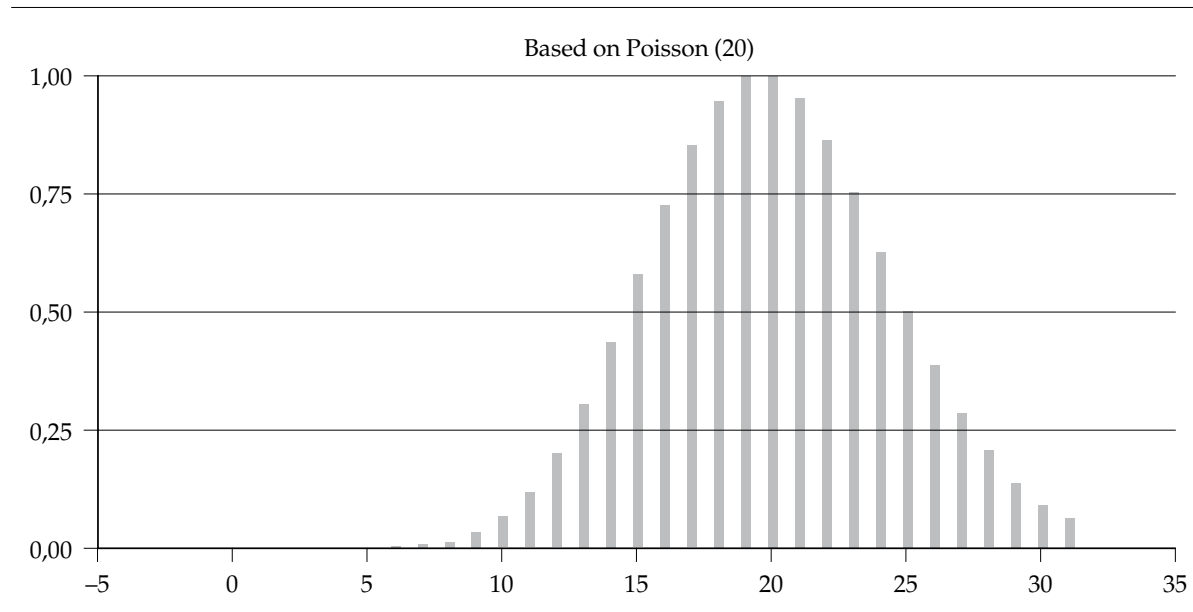

Figura 10. Distribución de número de reclamaciones con deducible $\$ 200.000 .000$

Fuente: elaboración de los autores empleando el programa @RISK. 


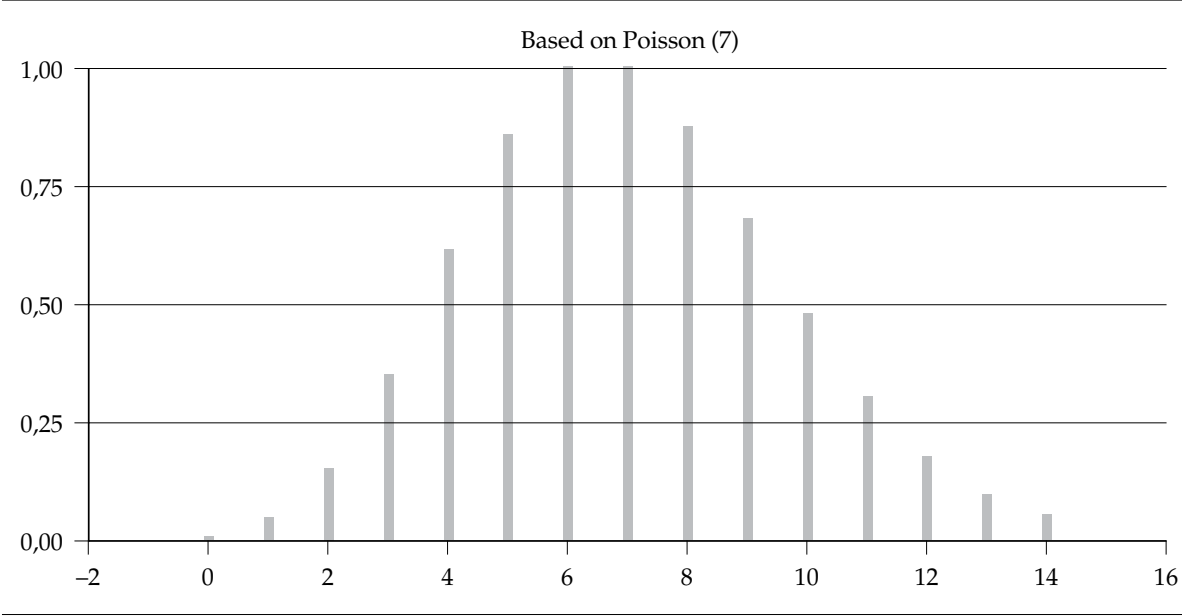

Figura 11. Distribución de número de reclamaciones con deducible $\$ 300.000 .000$

Fuente: elaboración de los autores empleando el programa @RISK.

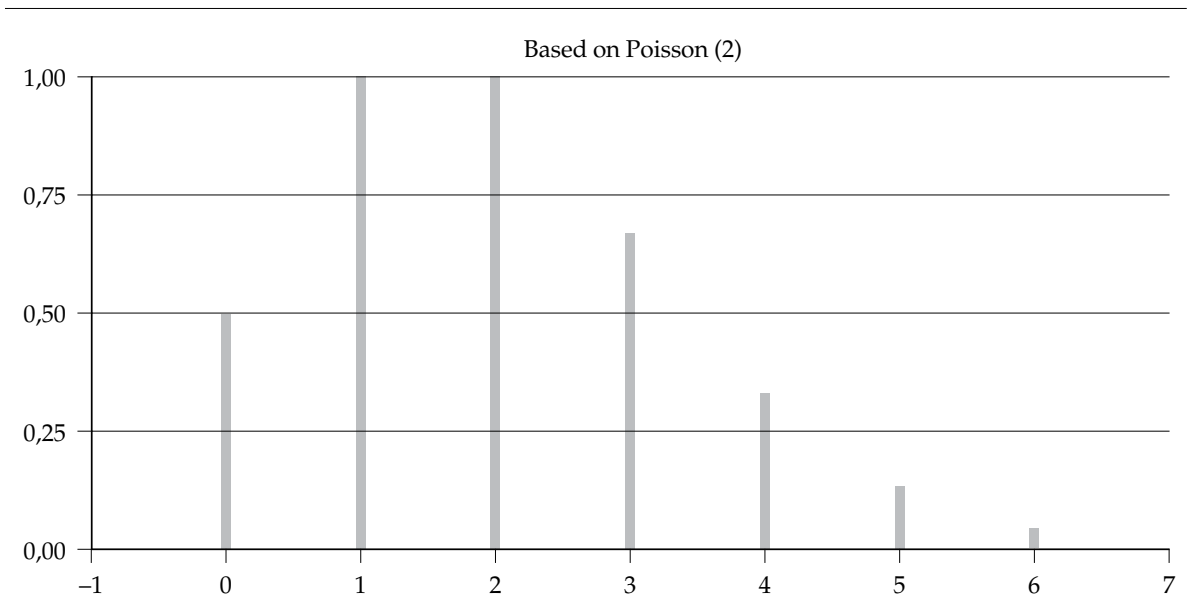

Figura 12. Distribución de número de reclamaciones con deducible $\$ 400.000 .000$

Fuente: elaboración de los autores empleando el programa @RISK. 


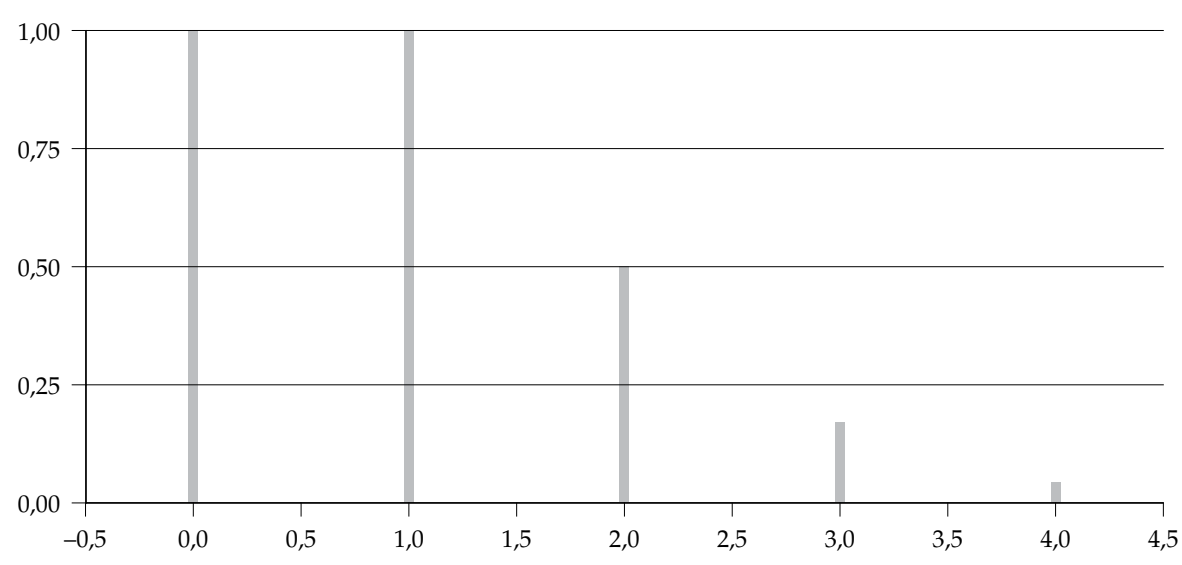

Figura 13. Distribución de número de reclamaciones con deducible $\$ 600.000 .000$ Fuente: elaboración de los autores empleando el programa @RISK.

La figura 14 ilustra qué tan cercanos se encuentran los datos estimados frente a los datos reales (montos de los siniestros), mediante una distribución log-normal. Entre más concentrados en la línea diagonal se encuentren, más ajustada es la distribución de probabilidad estimada a la realidad.

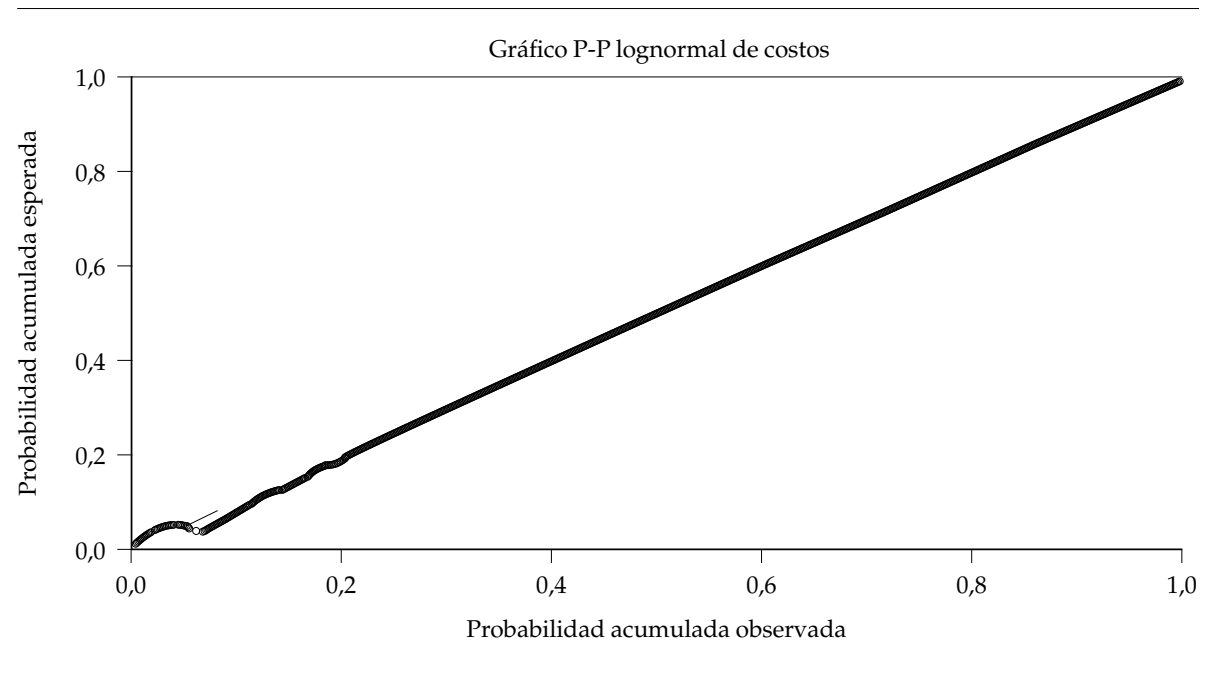

Figura 14. P-P log-normal de montos de siniestros

Fuente: elaboración de los autores empleando el programa SPSS. 

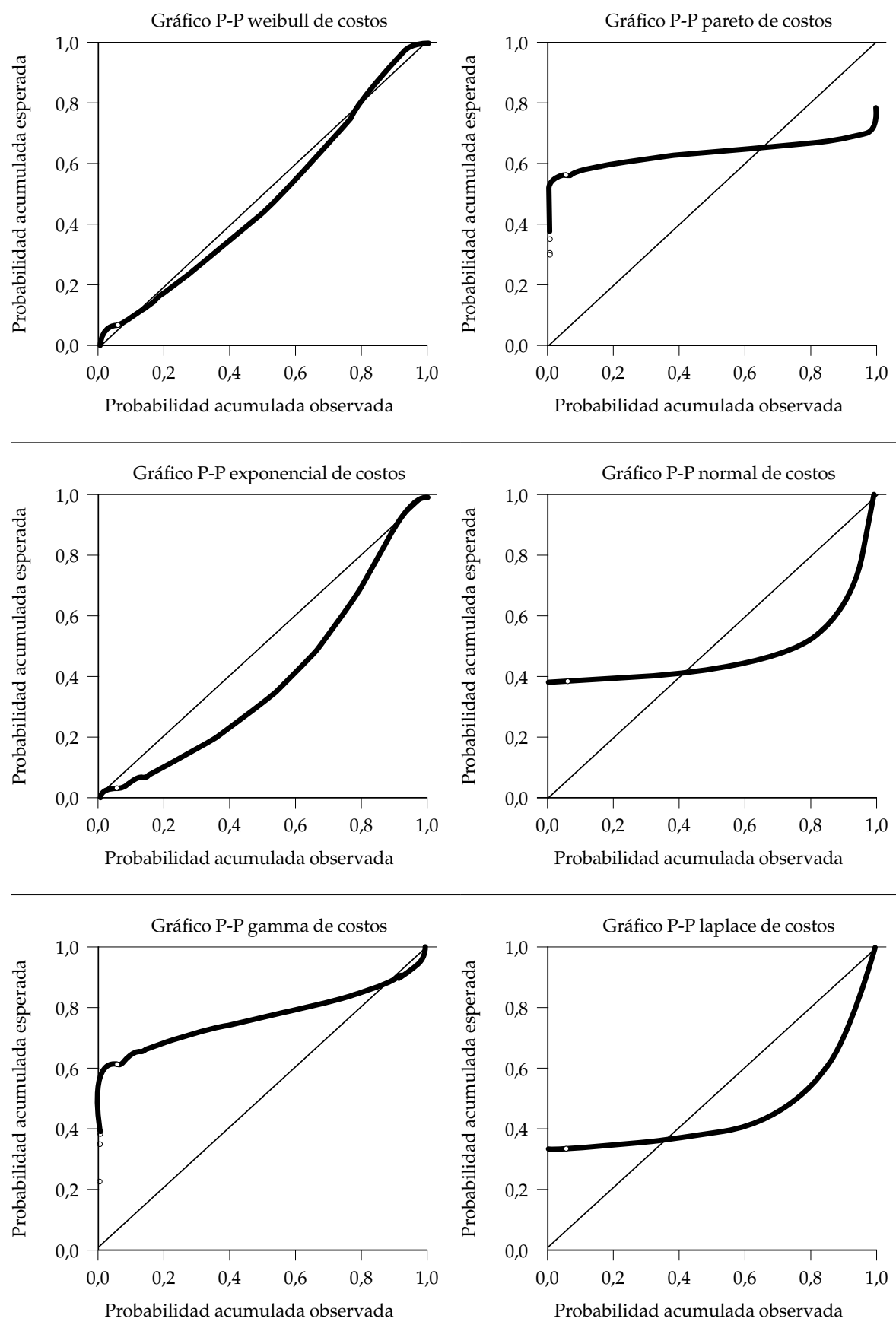

Figura 15. P-P otras distribuciones de montos de siniestros

Fuente: elaboración de los autores empleando el programa spss. 
Se puede observar que las anteriores distribuciones de probabilidad no se acercan a la aproximación realizada por la función log-normal. Para todo, luego de varias pruebas, se infirió que la distribución que mejor se ajusta a los datos es la distribución log-normal. Con este supuesto, el paso siguiente es estimar los parámetros de la función de distribución.

Primero se hace una estimación preliminar utilizando el método de momentos y luego se realizan las correspondientes pruebas de bondad de ajuste. El modelo de distribución de pérdida log-normal tiene la función de distribución de probabilidad dada por: $F(X)=\Phi(Z)$, con parámetros $\mu$ y $\sigma$; donde: $Z=\frac{\ln (X)-\mu}{\sigma}$.

Función de densidad de probabilidad dada por:

$$
F(X)=\frac{\Phi(Z)}{\sigma X}=\frac{1}{X \sigma \sqrt{2 \pi}} e^{\frac{-Z^{2}}{2}}
$$

El valor de los parámetros $\mu$ y $\sigma$ son los que diferencian cada función de distribución. Al utilizar el método de momentos, se sabe que $m_{1}=e^{\mu+\sigma^{2} / 2}$ y que $m_{2}=e^{2 \mu+4 \sigma^{2} / 2}$. Por lo tanto:

$$
\begin{gathered}
\sigma=\sqrt{\ln \left(m_{2}\right)-2 * \ln \left(m_{1}\right)} \\
\mu=\ln \left(m_{2}\right)-\frac{\sigma^{2}}{2}
\end{gathered}
$$

Donde:

$m_{k}=E\left(x^{k}\right)$ es el momento de orden $K$.

Como:

$$
m_{k}=E\left(x^{k}\right)=\sum_{i=1}^{n} \frac{x_{i}^{k}}{n}
$$

Realizando las operaciones correspondientes, se encuentra que los valores de los parámetros son: 


$$
\begin{aligned}
& \mu=13,027 \\
& \sigma=1,571
\end{aligned}
$$

Ahora se procede a realizar las pruebas de bondad de ajuste, con la prueba de ji-cuadrado.

$H_{o}=$ la distribución del conjunto de datos se aproxima a la distribución log-normal.

$H_{1}=$ el modelo no responde a la distribución log-normal.

Dado el coeficiente ji-cuadrado: $X_{G}^{2}(n)$

Donde:

$n=$ Grados de libertad;

$G=$ Nivel de significancia .

Se busca que la prueba tenga un nivel de significancia del $5 \%$ y que tenga 19 grados de libertad, ya que son 19 los intervalos de clase que se tienen (regla de Sturges). Es decir, el coeficiente ji-cuadrado, que corresponde en la tabla a $X_{0.05}^{2}(19)$ es: $C=28,87$.

Ahora se procede a calcular el coeficiente utilizando las frecuencias observadas y las frecuencias esperadas. Así, se tiene que:

$$
Q=\sum \frac{f_{0}-f_{e}}{f_{e}}=17,53
$$

Donde:

$f_{0}=$ frecuencia observada

$f_{e}=$ frecuencia esperada

Como $Q<C$, entonces se acepta la hipótesis nula $H_{0}$. De esta manera se determina que la función de distribución de los montos de siniestros sigue el modelo de distribución log-normal.

Ya teniendo identificadas las funciones de distribución del número de siniestros y del monto de este, se aplica el modelo descrito en la sección 3.1.

$$
P=\sum_{n=1}^{\infty} p(n) \int_{0}^{\infty} x S^{* n}(x) d x
$$


Para ello se usa el aplicativo @RISK de función compuesta, modelo severidad-frecuencia.

A continuación se presentan los resultados obtenidos al aplicar el modelo actuarial mediante dos vías: @RISK ponderado y SPSS. Comparando los resultados con el método determinístico (empírico), en el que no se utiliza ninguna distribución de probabilidad, en donde el cálculo de la prima es muy sencillo, pues simplemente suma el total de los costos que superaron el deducible, para luego dividirlo sobre el total de la población expuesta al riesgo. Se obtiene entonces de esta forma un costo per capita.

Tabla 6. Primas puras de riesgo reaseguro - modelo actuarial

\begin{tabular}{|c|c|c|c|c|c|c|c|}
\hline \multirow{2}{*}{$\begin{array}{l}\text { Método } \\
\text { utilizado }\end{array}$} & \multicolumn{7}{|c|}{ Deducible } \\
\hline & $\$ 50$ mill. & $\$ 75$ mill. & $\$ 100$ mill. & $\$ 200$ mill. & $\$ 300$ mill. & $\$ 400$ mill. & $\$ 600$ mill. \\
\hline $\begin{array}{l}\text { Determinístico } \\
\text { (empírico) }\end{array}$ & $\$ 60.292$ & $\$ 39.506$ & $\$ 27.542$ & $\$ 9.440$ & $\$ 4.184$ & $\$ 2.160$ & $\$ 259$ \\
\hline $\begin{array}{l}\text { Probabilístico } \\
\text { usando @RISK } \\
\text { (ponderado) }\end{array}$ & $\$ 60.427$ & $\$ 47.602$ & $\$ 37.795$ & $\$ 20.126$ & $\$ 10.512$ & $\$ 3.962$ & $\$ 2.932$ \\
\hline $\begin{array}{l}\text { Probabilístico } \\
\text { usando sPSS y } \\
\text { @RISK }\end{array}$ & $\$ 57.719$ & $\$ 46.124$ & $\$ 36.926$ & $\$ 19.897$ & $\$ 10.426$ & $\$ 3.936$ & $\$ 2.921$ \\
\hline
\end{tabular}

Fuente: elaboración de los autores.

Los resultados muestran una relación directa para el método actuarial, comparando las funciones predeterminadas en los softwares @RISK y SPSS.

\subsection{Resultados del modelo Black-Scholes}

Los parámetros tomados para cada variable del modelo de Black-Scholes son los siguientes:

\subsubsection{Volatilidad}

$$
\sigma=0,335
$$

Resultado de multiplicar la desviación estándar de la variación (retornos diarios) del valor total de cuentas asistenciales radicadas en la EMP (1,95\%) por la raíz cuadrada del número de días, en el año, en que se radicaron cuentas (296 días). Este valor se asume igual para cada uno de los afiliados a la EMP. 
4.3.2. Precios de los activos subyacentes

$$
S_{0}=\$ 100 ; \ldots ; \$ 582.967 .235^{2}
$$

Es el valor total del costo registrado en el año por cada uno de los afiliados a la EMP. Los valores oscilaron entre cien pesos (\$100) y seiscientos millones de pesos $(\$ 600.000 .000)$.

\subsubsection{Tasa de interés}

$$
r=4,19 \%
$$

Se toma como referencia la tasa de interés de los TES de Colombia, con vencimiento 2014 y cierre del 10 de octubre de 2013.

\subsubsection{Plazo de ejercicio}

$$
t=1
$$

El período de vigencia de los reaseguros en salud es generalmente de un año. Los valores obtenidos para la call mediante la fórmula de Black-Scholes para cada uno de los afiliados se suman, y luego se dividen por el total de la población, para tener así el valor promedio de la prima por individuo. Esto se realiza teniendo en cuenta que la reaseguradora cobrará una prima única por afiliado. Se presentan varios escenarios de acuerdo con el deducible o precio de ejercicio $(E)$. Las primas presentadas son anuales.

Tabla 7. Primas puras de riesgo reaseguro - modelo opción call Black-Scholes

\begin{tabular}{lccccccc}
\hline \multicolumn{7}{c}{ Deducible o precio de ejercicio $(E)$} \\
\hline $\begin{array}{l}\text { Opción call } \\
\text { europea B-S }\end{array}$ & $\$ 50$ mill. & $\$ 75$ mill. & $\$ 100$ mill. & $\$ 200$ mill. & $\$ 300$ mill. & $\$ 400$ mill. & $\$ 600$ mill. \\
\hline
\end{tabular}

Fuente: elaboración de los autores.

\subsection{Comparación de resultados}

A las primas puras de riesgo anteriormente estimadas se hace necesario incluirles un factor de seguridad relativo $\theta$, que busca proteger al reasegurador

2 Total de afiliados que generaron costo: 201.505 . 


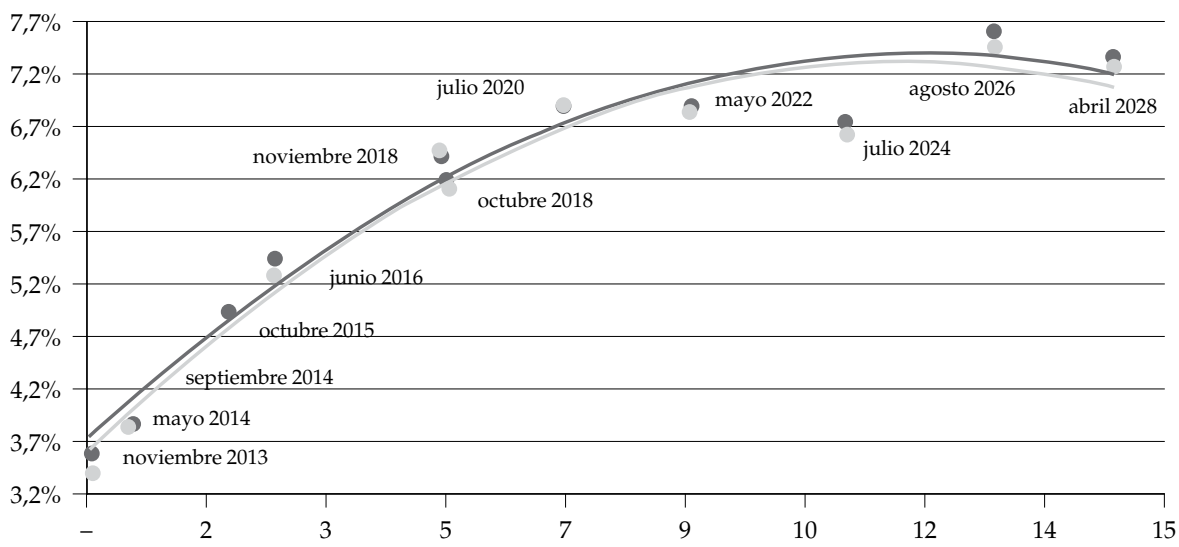

Años restantes para vencimiento

Cierre 30 sep. 2013 Cierre 10 oct. 2013

\begin{tabular}{lcc}
\hline \multicolumn{1}{c}{ TES } & Cierre 30 sep. 2013 & Cierre 10 oct. 2013 \\
\hline Noviembre 2013 & $3,60 \%$ & $3,39 \%$ \\
Mayo 2014 & $3,89 \%$ & $3,84 \%$ \\
Septiembre 2014 & $4,21 \%$ & $4,19 \%$ \\
Octubre 2015 & $4,92 \%$ & $4,77 \%$ \\
Junio 2016 & $5,44 \%$ & $5,28 \%$ \\
Octubre 2018 & $6,43 \%$ & $6,49 \%$ \\
Noviembre 2018 & $6,19 \%$ & $6,12 \%$ \\
Julio 2020 & $6,90 \%$ & $6,91 \%$ \\
Mayo 2022 & $6,89 \%$ & $6,85 \%$ \\
Julio 2024 & $6,74 \%$ & $6,65 \%$ \\
Agosto 2026 & $7,61 \%$ & $7,47 \%$ \\
Abril 2028 & $7,37 \%$ & $7,27 \%$ \\
\hline
\end{tabular}

Figura 16. Tasa TES de Colombia

Fuente: Tesorería Banco de Bogotá - SEN - página web del Grupo Aval.

ante desviaciones de siniestralidad (Herzog, 1994; Bailey, 1950). Este valor es calculado bajo la siguiente fórmula:

$$
\theta=\frac{Z \times \sqrt{V\left(\operatorname{Ln}\left(\operatorname{Costo}_{i}\right)\right)}}{E\left(\operatorname{Ln}\left(\operatorname{Costo}_{i}\right)\right)}
$$


Donde: $Z$ es el percentil de la distribución normal estándar, el cual se asu-

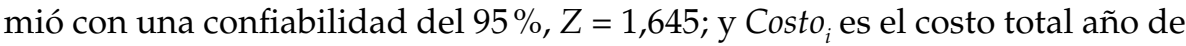
cada afiliado.

$$
\theta=13,79 \%
$$

Por lo tanto, la prima con factor de seguridad se calcula como sigue:

$$
\text { PrimaNeta }=\text { PrimaPuradeRiesgo } *(1+\theta)
$$

Tabla 8. Primas con factor seguridad - comparativo modelo actuarial y opción call Black-Scholes

\begin{tabular}{lccccccc}
\hline \multirow{2}{*}{ Método utilizado } & \multicolumn{7}{c}{ Deducible } \\
\cline { 2 - 7 } & $\$ 50$ mill. & $\$ 75$ mill. & $\$ 100$ mill. & $\$ 200$ mill. & $\$ 300$ mill. & $\$ 400$ mill. & $\$ 600$ mill. \\
\hline $\begin{array}{l}\text { Determinístico (empírico) } \\
\begin{array}{l}\text { Actuarial usando @risk } \\
\text { (ponderado) }\end{array}\end{array}$ & $\$ 68.605$ & $\$ 44.953$ & $\$ 31.339$ & $\$ 10.742$ & $\$ 4.761$ & $\$ 2.457$ & $\$ 295$ \\
$\begin{array}{l}\text { Actuarial usando sPSS y } \\
\begin{array}{l}\text { @risk } \\
\text { Opción call europea B-S }\end{array}\end{array}$ & $\$ 68.758$ & $\$ 54.165$ & $\$ 43.006$ & $\$ 22.900$ & $\$ 11.961$ & $\$ 4.509$ & $\$ 3.337$ \\
\hline
\end{tabular}

Fuente: elaboración de los autores.

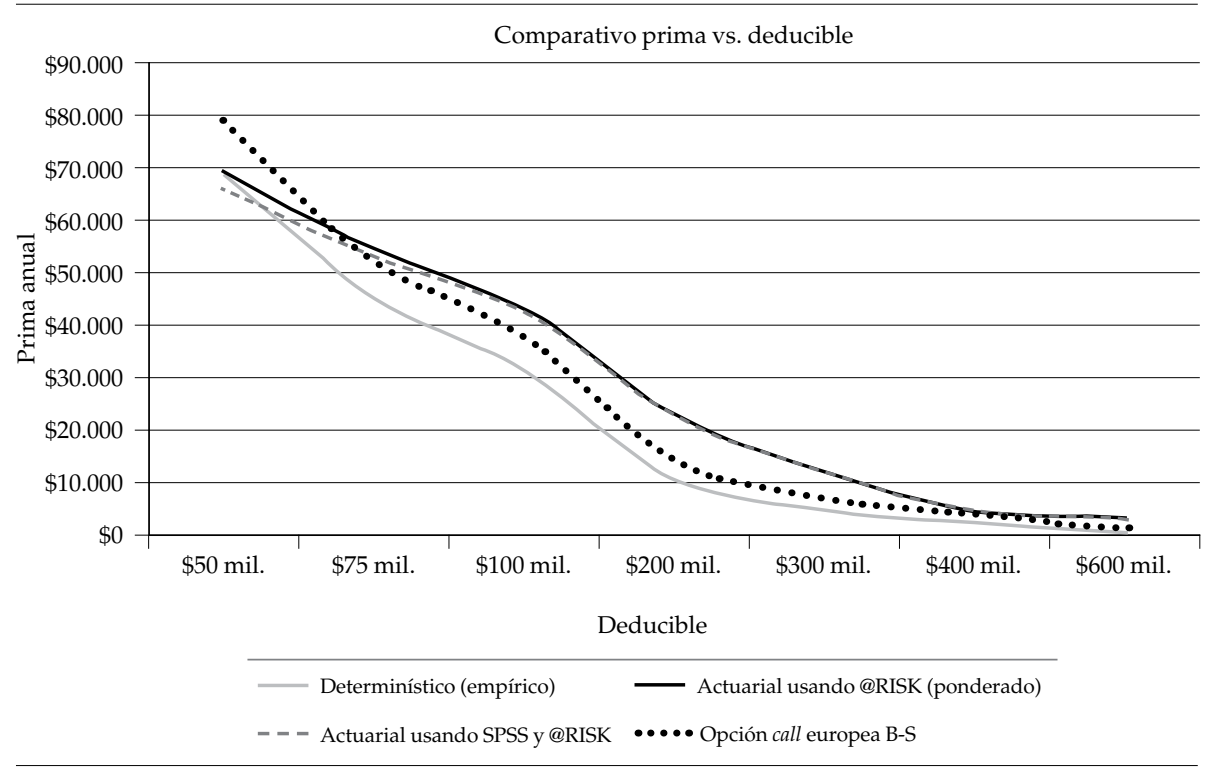

Figura 17. Comparativo primas comerciales modelo actuarial, empírico y opción call Black-Scholes

Fuente: elaboración de los autores. 
Se observa cómo, con un deducible de $\$ 75$ millones, las primas del reaseguro en cada uno de los modelos se aproximan considerablemente, y se diferencian bastante cuando el deducible es mayor a \$200 millones.

\section{Conclusiones y recomendaciones}

La conjetura principal de cualquier contraste sobre los modelos de valoración de opciones es saber si la distribución de probabilidad que denota los valores del activo subyacente es consistente con la distribución dada por el modelo teórico de valoración. La presente investigación demuestra que el valor del activo subyacente utilizado obedece a una distribución log-normal; situación que hace expedita la aplicación del modelo de Black-Scholes para valorar opciones.

De acuerdo con los resultados obtenidos, se demuestra que el modelo de Black-Scholes, que se utiliza normalmente para valorar opciones financieras, puede ser también utilizado para la estimación de primas de reaseguros de salud, y que la prima neta estimada a partir de este modelo se aproxima a las establecidas por el método actuarial, excepto cuando el deducible del reaseguro es muy alto (por encima de $\$ 200$ millones). Esto se debe a que el número de eventos esperados, o siniestros susceptibles a reclamo, es muy bajo a medida que aumenta el deducible y, por ende, el riesgo (incertidumbre) es mayor.

Este acercamiento de las primas obtenidas por los dos modelos permite incorporar una nueva metodología en el cálculo de la prima de reaseguramiento, menos sofisticada con relación a los métodos actuariales. De esta forma, se enriquecen los modelos de reaseguramiento de enfermedades de alto costo utilizados por las EMP. Es importante aclarar que cualquier adaptación que se quiera realizar del modelo planteado, siguiendo el esquema de opciones con valoración Black-Scholes, requerirá disponer de suficiente información histórica, principalmente para la estimación de la volatilidad.

La equivalencia entre la adquisición de opciones y las operaciones de reaseguro hace que la teoría de valoración de opciones se perfile como un buen método alternativo utilizable para estimar el valor de las primas de reaseguramiento en salud. La posibilidad de reproducir una operación de reaseguro en el mercado de las EMP, a través de un sintético, abren el camino a la utilización de la teoría de valoración de opciones para el cálculo de primas del reaseguro.

Se es consciente de que existen muchas investigaciones que han demostrado que los retornos del subyacente no siempre son normales, o que los precios del subyacente no son log-normales. Aunque en el presente trabajo queda demostrado que el valor del subyacente se distribuye log-normal. Sin embargo, en el contexto financiero se ha probado que la log-normalidad no siempre se 
da, por ejemplo, con distribuciones de colas más pesadas, leptocúrticas, como la distribución $t$.

El modelo de Black-Scholes supone constante la volatilidad de todas las opciones sobre el mismo subyacente y la misma fecha de vencimiento, pero diferentes precios de ejercicio deberían tener la misma volatilidad. Sin embargo, en los mercados reales existe amplia evidencia con hechos estilizados sobre la denominada sonrisa de volatilidad. Por ejemplo, el patrón de la volatilidad implícita en relación con el grado del dinero ${ }^{3}$ de cada opción (Marín y Rubio, 2001).

En el ejercicio aplicado se encontró que, cuando el deducible del reaseguro es muy alto, la prima de la call es más baja que la estimada por el modelo actuarial, explicado por la existencia de muy pocas opciones in the money en el agregado. Ocurre lo contrario cuando el deducible es más bajo; el modelo de Black-Scholes se acerca al modelo actuarial, explicado porque se tienen más opciones in the money en el agregado.

Cuando la sonrisa de volatilidad es simétrica, Black-Scholes infravalora opciones call tanto in the money como out the money. De hecho, una sonrisa simétrica refleja que la distribución del subyacente presenta colas más gruesas que la normal tanto a la derecha como a la izquierda y, por lo tanto, sugiere la presencia de exceso de curtosis. Si la distribución de probabilidades tuviese la cola izquierda más gruesa pero cola derecha más estrecha que la normal, Black-Scholes infravaloraría opciones call in the money y sobrevaloraría la call out the money. Esto es lo que ocurre con sonrisas asimétricas con pendiente negativa, como lo menciona Marín y Rubio (2001).

Los modelos de valoración de opciones que pretendan valorar más rigurosamente los precios de las opciones podrían incorporar estos tipos de comportamiento en su estructura teórica. Además, lograrían ser modelos capaces de capturar correlaciones tanto negativas como positivas entre la volatilidad y el subyacente. Los paradigmas que se han desarrollado en la literatura reciente admiten posibilidades de modelar explícitamente saltos en el subyacente, o introducir comportamientos concretos en la volatilidad estocástica de este. Por ejemplo, el modelo de Heston (1993) puede mejorar sensiblemente el comportamiento del modelo de Black-Scholes. Evidentemente, el modelo será más rico que el de Black-Scholes, pero es necesario estimar un mayor número de parámetros.

3 El grado del dinero se define como el cociente $K / P$, donde $P$ es el precio del subyacente que se hace igual para todas las opciones empleadas en la estimación. 


\section{Referencias}

Bailey, A. (1950). Credibility procedures: Laplace's generalization of Bayes rule and the combination of collateral knowledge with observed data. Proceedings of the Casualty Actuarial Society, 37, 7-23.

Berger, J. O. (1985). Statistical decision theory and bayesian analysis. New York: Springer.

Black, F., \& Scholes, M. (1973). The pricing of options and corporate liabilities. Journal of Political Economy, 81, 637-654.

Bowers et al. (1997). Actuarial mathematics. Itasca, IL: Society of Actuaries.

Brennan, M., \& Schwartz, E. (1976). The pricing of equity-linked life insurance policies with an asset value guarantee. Journal of Financial Economics, 3, 195-213.

Chicaíza, L. (2002). El sistema de aseguramiento en salud de Colombia: estimación de primas de reaseguro utilizando la teoría de valoración de opciones (Tesis doctoral, Universidad Politécnica de Valencia).

Chicaíza, L. (2003). Valoración de primas de reaseguro para enfermedades catastróficas Black-Scholes. Universidad Externado de Colombia, Facultad de Economía.

Cummins, H., \& Geman, J. (1995). Pricing catastrophe insurance futures and call spreads: an arbitrage approach. The Journal of Fixed Income, 4(4), 46-57.

Deelstra, G., \& Platin, G. (2014). Risk theory and reinsurance. London: SpringerVerlag.

De Vydler, E. (1996). Advanced risk theory. Bruxelles: Université de Bruxelles.

Demeterfi, K., Derman, E., Kamal, M., \& Zou, J. (1999). A guide to volatility and variance swaps. Journal of Derivative, 6(4), 9-32.

Embrechts, P. (1996). Actuarial versus financial pricing of insurance. Department of Mathematics, Swiss Federal Institute of Technology Zurich.

Embrechts, P., \& Meister, S. (1997). Pricing insurance derivatives, the case of CAT futures. Securitization of Insurance Risk: The 1995 Bowles Symposium.

Gerber, H. \& Shiu, E. (1994). Option pricing by Esscher transforms. Transactions of Society of Actuaries, 46.

Guyon, J., \& Labordère, P. (2014). Nonlinear option pricing. Taylor \& Francis Group, LLC.

Herzog, T. (1994). Introduction to credibility theory. Michigan: Actex Publications.

Heston, S. (1993). A closed-form solution for options with stochastics volatility and applications to bond and currency options. Review of Financial Studies, 3, 267-286.

Hogg, R., \& Klugman, S. (1979). Loss distributions. New York: Jhon Wiley and Sons. 
Kawamoto, B. (1998). Insurance linked derivatives. Financing Risk \& Reinsurance, 7 .

Lamothe, P., \& Pérez, M. (2006). Opciones financieras y productos estructurados ( $3^{\mathrm{a}}$ ed.). Madrid: McGraw-Hill.

Lundberg, O. (1940). On random processes and their application to sickness and accident statistics (Tesis doctoral, University of Stockholm).

Marín, J., \& Rubio, G. (2001). Economía financiera. Barcelona: Bosch Editor.

Merton, R. C. (1977). An analytic derivation of the cost of deposit insurance and loan guarantees: an application of modern option pricing theory. Journal of Banking and Finance, 1, 3-11.

Mood, A. M., Graybill, F. A., \& Boes, D. C. (1974). Introduction to the theory of statistics. Singapur: McGraw-Hill.

Petersen, M. A., \& Thiagarajan, S. R. (2000). Risk measurement and hedging: with and without derivatives. Financial Management, 29(4), 5-29.

Phillips, R. D., Cummins, J. D., Allen, F. (1998). Financial pricing of insurance in a multiline company. The Journal of Risk and Insurance, 65(4), 597-636.

Qiu, J., Li, M., Wang, Q., \& Wang, B. (2012). Catastrophe reinsurance pricing: science, art or both? Recuperado de file:/ / C:/Users/legiron/Downloads/ Emphasis-2-2012-Catastrophe-Reinsurance-Pricing.pdf

Shreve, S. E. (2004). Stochastic for finance II. Continuos time models. New York: Springer Finance Series.

Torres, A., \& Agudelo, D. (2001). Simulación financiera, organización y gestión aplicada a la valoración de opciones reales. Caso colombiano: Medellín. 